$\underline{\text { Preprint typeset in JHEP style - PAPER VERSION }}$

hep-lat/0210017

DPNU-02-32

CERN-TH/2002-254

\title{
Lattice Perturbation Theory in Noncommutative Geometry and Parity Anomaly in 3D Noncommutative QED
}

\author{
Jun Nishimura \\ Department of Physics, Nagoya University \\ Furo-cho, Chikusa-ku, Nagoya 464-8602, Japan \\ E-mail: nisimura@eken.phys.nagoya-u.ac.jp \\ Miguel A. Vázquez-Mozo \\ Theory Division, CERN \\ CH-1211 Geneva 23, Switzerland \\ E-mail: Miguel.Vazquez-Mozo@cern.ch
}

\begin{abstract}
We formulate lattice perturbation theory for gauge theories in noncommutative geometry. We apply it to three-dimensional noncommutative QED and calculate the effective action induced by Dirac fermions. In particular "parity invariance" of a massless theory receives an anomaly expressed by the noncommutative Chern-Simons action. The coefficient of the anomaly is labelled by an integer depending on the lattice action, which is a noncommutative counterpart of the phenomenon known in the commutative theory. The parity anomaly can also be obtained using Ginsparg-Wilson fermions, where the masslessness is guaranteed at finite lattice spacing. This suggests a natural definition of the lattice-regularized Chern-Simons theory on a noncommutative torus, which could enable nonperturbative studies of quantum Hall systems.
\end{abstract}

Keywords: Lattice Gauge Field Theories, Non-Commutative Geometry, Anomalies in Field and String Theories, Chern-Simons Theories. 


\section{Contents}

1. Introduction 1

2. Lattice perturbation theory in noncommutative geometry 3

2.1 Noncommutative QED on the lattice 3

$\begin{array}{lll}2.2 & \text { Feynman rules } & 7\end{array}$

2.3 Perturbative evaluation of the effective action 8

3. The parity anomaly in 3D noncommutative QED 9

3.1 A brief review of the commutative case 10

3.2 The noncommutative case 11

3.2.1 Fundamental fermions 11

$\begin{array}{lll}3.2 .2 & \text { Adjoint fermions } & 14\end{array}$

4. Noncommutative Chern-Simons theory on the lattice 18

5. Concluding remarks 20

$\begin{array}{ll}\text { A. Functional determinants } & 21\end{array}$

B. Evaluation of the Feynman integrals $\quad 24$

B.1 Fundamental fermions 24

B.2 Adjoint fermions 25

\section{Introduction}

Quantum field theory on noncommutative spaces has been a subject of much activity in recent years (see $[1,2]$ for comprehensive reviews). This attention was originally triggered by its intimate relationship with string theory, but the study of noncommutative field theories has interest in its own. From a mathematical physics point of view they provide us with a class of nonlocal quantum field theories which nonetheless seem to be well defined. Also, since noncommutative field theories are essentially theories of dipoles [3] they can be also useful in the analysis of systems with dipolar excitations in condensed matter physics.

In this context, noncommutative Chern-Simons (NCCS) theory in $(2+1)$-dimensions is specially appealing because of its applications to quantum Hall systems $[4,5]$. Here the noncommutativity is introduced only in the spatial direction and the resulting deformation of the ordinary gauge invariance into "star-gauge invariance" is essential for the description of the system which has the area preserving diffeomorphism invariance [4]. Remarkably, a 
finite- $N$ matrix model of NCCS theory is found to have physical states which have one-toone correspondence with Laughlin-type wave functions [6]. It has also been pointed out that instabilities of the NCCS theory can describe the transition to the Wigner crystal, where spontaneous breakdown of translational invariance is caused by the noncommutativity [7].

In studying noncommutative field theories it is often useful to consider its matrixmodel description. This is reminiscent of its string/M-theoretic connections [8, 9]. Here the space-time degrees of freedom and the internal ("color") degrees of freedom are treated on equal footing, and "star-gauge invariance" is simply described by the global $\mathrm{U}(\infty)$ symmetry which acts on the matrix indices. The matrix model description is also useful for regularizing noncommutative field theories $[9,10,11,12,13]$, since finite- $N$ twisted reduced models [14] are interpreted as a lattice formulation of noncommutative field theories [11, $12,13]$. Such a lattice formulation provides the most reliable method to study the quantum dynamics of noncommutative field theories in a fully nonperturbative manner. Recently, the lattice regulatization has been applied to two-dimensional noncommutative Yang-Mills theory $[15,16]$, where (nonperturbative) renormalizability was demonstrated for the first time in a noncommutative field theory (see also [17]). There the same theory was shown to have an intriguing infrared property which may be described as the Aharonov-Bohm effect with the magnetic field identified with the inverse noncommutativity parameter. The lattice formulation has also been used to explore the phase diagram of noncommutative scalar field theories [16, 18], which is expected to be richer than in the commutative case, as indicated by a self-consistent Hartree approximation [19]. In particular, as conjectured by Ref. [19], the ordered phase is found to split into a uniformly ordered phase and a phase dominated by the stripe pattern. In the latter phase, nonzero momentum modes acquire vacuum expectation values, and therefore translational invariance is spontaneously broken.

The aim of this paper is two-fold. First we formulate perturbation theory for the lattice noncommutative gauge theory. Although the most important virtue of the lattice regularization lies in its capability of nonperturbative studies, it has also been used to clarify subtle issues in perturbative aspects of gauge theories. We consider this particularly important because the lattice construction of noncommutative chiral gauge theories suggests a new mechanism of gauge anomaly cancellation, which is not yet known in the continuum [20]. As an application of the lattice perturbation theory, we pick up a noncommutative version of three-dimensional $\mathrm{QED}$, where the lattice calculation indeed plays a crucial role in revealing peculiar properties of the parity anomaly, given in terms of noncommutative Chern-Simons action. The coefficient of the anomaly is labelled by an integer depending on the lattice action, which is a counterpart of the phenomenon observed by Coste and Lüscher [21] in the commutative theory. The commutative limit is smooth when the fermions are in the fundamental representation, but it is not for fermions in the adjoint representation due to a characteristic property of noncommutative field theories known as the UV/IR mixing [22]. As a special case our result includes Chu's result [23] obtained in the continuum by using the Pauli-Villars regularization.

Another aim of this work is to construct a lattice regularization of NCCS theory, which has important applications to quantum Hall systems as mentioned above. In general, defining a Chern-Simons term on the lattice is not straightforward due to its topological nature. 
A recent proposal [24] is to utilize Ginsparg-Wilson fermions in odd dimensions and to define the Chern-Simons term using the parity violating part of the effective action induced by the fermion. We show that this proposal works also in the noncommutative case. As we mentioned above, the parity anomaly in the three-dimensional noncommutative QED is given by the NCCS term in the continuum limit of the lattice theory we started with. The same result can be obtained from Ginsparg-Wilson fermions, where the masslessness is guaranteed at finite lattice spacing. This suggests a natural definition of the lattice-regularized Chern-Simons theory on a noncommutative torus, which could enable nonperturbative studies of quantum Hall systems. In this regard, we recall that a finite- $N$ matrix model has been proposed as a regularized description of NCCS theory on a cylinder [25]. Although our theory can also be mapped to a finite- $N$ matrix model, the two proposals appear to be quite different.

The rest of the paper is organized as follows. In Section 2, we review the lattice formulation of noncommutative gauge theories and formulate a perturbation theory based on Feynman rules. In Section 3, we present calculations of the parity anomaly in noncommutative QED. In Section 4 we discuss the parity anomaly using Ginsparg-Wilson fermions, which leads to a proposal for a lattice-regularized noncommutative Chern-Simon theory. Section 5 is devoted to summary and discussions. Finally, in Appendix A a calculation of the parity-violating terms in the effective action by the direct evaluation of the fermionic determinant is presented, while Appendix B contains some details of the computation of the Feynman integrals involved in the diagrammatic calculation of Section 3.

\section{Lattice perturbation theory in noncommutative geometry}

In this section we begin by reviewing the lattice formulation of noncommutative gauge theories developed in Ref. [11, 12, 13] (see [26, 27, 28] for reviews). In the literature it is common to start from a finite- $N$ matrix model, which is then shown to be equivalent to the lattice formulation of a noncommutative field theory. Indeed, the matrix model representation has proven useful for numerical analyses $[15,16,18,20]$. Here we will work directly with the lattice formulation and derive the Feynman rules, which are used in the perturbative evaluation of the effective action induced by fermions. Although we will focus on noncommutative QED in $d=2 m+1$ dimensions, the lattice perturbation theory can be formulated for any other noncommutative field theories in the same way.

\subsection{Noncommutative QED on the lattice}

In noncommutative geometry, space-time coordinates are treated as Hermitian operators obeying the commutation relation $\left[\hat{x}_{\mu}, \hat{x}_{\nu}\right]=i \theta_{\mu \nu}$, where $\theta_{\mu \nu}$ is a real anti-symmetric matrix. In $(2 m+1)$ dimensions, due to a property of antisymmetric matrices, coordinates can always be chosen in such a way that one of them commutes with all the others, resulting in

$$
\left[\hat{x}_{i}, \hat{x}_{j}\right]=i \theta_{i j}, \quad\left[\hat{x}_{i}, \hat{x}_{d}\right]=0, \quad(i, j=1, \ldots, 2 m),
$$


where, for simplicity, the $2 m \times 2 m$ noncommutative matrix $\theta_{i j}$ is taken to be of the form

$$
\theta_{i j} \equiv \theta \varepsilon_{i j}, \quad \varepsilon=\left(\begin{array}{ccccc}
0 & -1 & & & \\
1 & 0 & & & \\
& & \ddots & & \\
& & & 0 & -1 \\
& & & 1 & 0
\end{array}\right) \text {. }
$$

We regard the commuting coordinate $\hat{x}_{d}$ as the Euclidean time after the Wick rotation. Field theories on a noncommutative geometry can be obtained by replacing an ordinary field $\phi(x)$ by an operator $\phi(\hat{x})$. An equivalent way to describe noncommutative field theories, which we are going to use in what follows, is to keep the ordinary field $\phi(x)$ but to replace the ordinary product of fields, say $\phi_{1}(x)$ and $\phi_{2}(x)$, by the star-product

$$
\phi_{1}(x) \star \phi_{2}(x)=\phi_{1}(x) \exp \left(\frac{i}{2} \theta_{\mu \nu} \overleftarrow{\partial_{\mu}} \overrightarrow{\partial_{\nu}}\right) \phi_{2}(x)
$$

In order to consider the lattice regularization of such theories, we introduce a $(2 m+1)$ dimensional toroidal lattice $\Lambda_{L, T}$ defined by

$$
\Lambda_{L, T}=\left\{\left(x_{1}, \ldots, x_{d}\right) \in a \mathbb{Z}^{d} \mid-a \frac{L_{\mu}-1}{2} \leq x_{\mu} \leq a \frac{L_{\mu}-1}{2}\right\},
$$

where $a$ is the lattice spacing and $L_{1}=L_{2}=\cdots=L_{2 m}=L, L_{d}=T$. We have assumed $L, T \in \mathbb{N}$ to be odd [13]. The dimensionful extent of the lattice is $\ell=a L$ in the $2 m$ spatial directions and $\tau=a T$ along the Euclidean time. The fields on the lattice are assumed to obey the periodic boundary condition in all directions ${ }^{1}$.

In order to construct a lattice counterpart of the star-product (2.3), we define the Fourier transform

$$
\tilde{\phi}(p)=a^{4} \sum_{x \in \Lambda_{L, T}} \phi(x) \mathrm{e}^{-i p \cdot x}
$$

where the lattice momentum $p$ is discretized as

$$
p_{\mu}=\frac{2 \pi n_{\mu}}{a L_{\mu}}, \quad n_{\mu} \in \mathbb{Z},
$$

and the Fourier modes $\tilde{\phi}(p)$ are periodic under $n_{\mu} \mapsto n_{\mu}+L_{\mu}$. Then the lattice star-product can be defined through its Fourier transform as

$$
\widetilde{\phi_{1} \star \phi_{2}}(p)=\frac{1}{a^{d} L^{d-1} T} \sum_{q} \exp \left\{-\frac{i}{2} \theta_{i j}(p-q)_{i} q_{j}\right\} \tilde{\phi}_{1}(p-q) \tilde{\phi}_{2}(q)
$$

where the noncommutativity parameter is taken to be

$$
\theta=\frac{1}{\pi} L a^{2} .
$$

\footnotetext{
${ }^{1}$ To formulate a finite-temperature field theory, the boundary condition in the time direction has to be taken anti-periodic for fermions.
} 
Here and henceforth we assume that the summation over a momentum is restricted to the Brillouin zone; namely $(2.6)$ with $-\left(L_{\mu}-1\right) / 2 \leq n_{\mu} \leq\left(L_{\mu}-1\right) / 2$.

The above lattice formulation naturally results from matrix model description of noncommutative field theories, and most importantly it preserves all the algebraic properties of the star-product. Moreover the definition (2.7) is consistent with the periodicity of the lattice momentum (2.6) due to (2.8). One can also rewrite this definition (2.7) in an integral form as

$$
\phi_{1}(\vec{x}, t) \star \phi_{2}(\vec{x}, t)=\frac{1}{L^{2 m}} \sum_{\vec{y}} \sum_{\vec{z}} \phi_{1}(\vec{y}, t) \phi_{2}(\vec{z}, t) \mathrm{e}^{-2 i\left(\theta^{-1}\right)_{i j}\left(x_{i}-y_{i}\right)\left(x_{j}-z_{j}\right)},
$$

where the summation over $\vec{y}$ and $\vec{z}$ is taken only over the spatial lattice. This expression is consistent with the periodicity of the fields again due to (2.8). As is clear from these observations, the lattice regularization of noncommutative field theories inevitably requires the noncommuting directions to be compactified in a particular way (2.8) consistent with the noncommutativity. This reflects the UV/IR mixing [22] at a fully nonperturbative level $[12,13]$.

Ultimately we have to take the continuum limit $a \rightarrow 0$, and the lattice size should be sent to infinity $L, T \rightarrow \infty$. These two limits should be taken more carefully in noncommutive field theories than in commutative ones because we have an extra scale parameter $\theta$ related to $a$ and $L$ by (2.8). In any case we have a hierarchy of the scales

$$
a \ll \sqrt{\theta} \ll \ell
$$

in the regularized theory. In order to obtain finite $\theta$, the physical extent of the spatial direction $\ell=a L$ should inevitably go to infinity. The extreme case $\theta \rightarrow 0$ is generally different from the commutative theory (where $\theta=0$ for finite $a$ ), as we see later in concrete examples. The limit $T \rightarrow \infty$ in the time direction can be taken as in commutative theories, and one can have arbitrary $\tau$ independently of $\theta$ and $\ell$.

The U(1) gauge fields can be put on the lattice by

$$
\begin{aligned}
U_{\mu}(x)= & \mathcal{P} \exp _{\star}\left(i g \int_{x}^{x+a \hat{\mu}} \mathcal{A}_{\mu}(s) \mathrm{d} s\right) \\
= & \sum_{n=0}^{\infty}(i g)^{n} \int_{x}^{x+a \hat{\mu}} \mathrm{d} \xi_{1} \int_{\xi_{1}}^{x+a \hat{\mu}} \mathrm{d} \xi_{2} \cdots \int_{\xi_{n-1}}^{x+a \hat{\mu}} \mathrm{d} \xi_{n} \\
& \mathcal{A}_{\mu}\left(\xi_{1}\right) \star \mathcal{A}_{\mu}\left(\xi_{2}\right) \star \cdots \star \mathcal{A}_{\mu}\left(\xi_{n}\right),
\end{aligned}
$$

where $\mathcal{A}_{\mu}(x)$ is the (real) gauge field in the continuum. The path-ordering is necessary even in the $\mathrm{U}(1)$ case, because of the noncommutativity arising from the star-product. Note also that $U_{\mu}(x)$ is not unitary, but it is "star-unitary",

$$
U_{\mu}(x) \star U_{\mu}(x)^{*}=U_{\mu}(x)^{*} \star U_{\mu}(x)=1 .
$$

The continuum gauge field $\mathcal{A}_{\mu}(x)$ transforms under the "star-gauge transformation" as

$$
\mathcal{A}_{\mu}(x) \mapsto g(x) \star \mathcal{A}_{\mu}(x) \star g(x)^{\dagger}-\frac{i}{g} g(x) \star \frac{\partial}{\partial x_{\mu}} g(x)^{\dagger}
$$


where $g(x)$ is also star-unitary. Under this transformation, the link field $U_{\mu}(x)$ defined by (2.11) transforms as

$$
U_{\mu}(x) \mapsto g(x) \star U_{\mu}(x) \star g(x+a \hat{\mu})^{*} .
$$

The lattice action for the gauge field is given by

$$
S_{\mathrm{G}}=-\beta \sum_{x \in \Lambda_{T, N}} \sum_{\mu \neq \nu} U_{\mu}(x) \star U_{\nu}(x+a \hat{\mu}) \star U_{\mu}(x+a \hat{\nu})^{*} \star U_{\nu}(x)^{*},
$$

which is invariant under star-gauge transformation (2.14).

The fermion action is defined by

$$
S_{\mathrm{F}}=a^{3} \sum_{x} \bar{\psi}(x) \star\left(D_{\mathrm{w}}-M\right) \psi(x)
$$

where $D_{\mathrm{w}}$ is the Dirac-Wilson operator

$$
D_{\mathrm{w}}=\frac{1}{2} \sum_{\mu=1}^{d}\left[\gamma_{\mu}\left(\nabla_{\mu}^{*}+\nabla_{\mu}\right)+r a \nabla_{\mu}^{*} \nabla_{\mu}\right] .
$$

The expression of the forward and backward covariant derivatives depends on the transformation properties of the fermion field. In the case where $\psi(x)$ transforms in the fundamental representation

$$
\psi(s) \mapsto g(x) \star \psi(x) ; \quad \bar{\psi}(x) \mapsto \bar{\psi}(x) \star g(x)^{*}
$$

they are given respectively by

$$
\begin{aligned}
\nabla_{\mu} \psi & =\frac{1}{a}\left[U_{\mu}(x) \star \psi(x+a \hat{\mu})-\psi(x)\right] \\
\nabla_{\mu}^{*} \psi & =\frac{1}{a}\left[\psi(x)-U_{\mu}(x-a \hat{\mu})^{*} \star \psi(x-a \hat{\mu})\right] .
\end{aligned}
$$

On the other hand, when fermions transform in the adjoint representation

$$
\psi(x) \mapsto g(x) \star \psi(x) \star g(x)^{*} ; \quad \bar{\psi}(x) \mapsto g(x) \star \bar{\psi}(x) \star g(x)^{*},
$$

the forward and backward covariant derivatives are respectively defined by

$$
\begin{aligned}
& \nabla_{\mu} \psi=\frac{1}{a}\left[U_{\mu}(x) \star \psi(x+a \hat{\mu}) \star U_{\mu}(x)^{*}-\psi(x)\right] \\
& \nabla_{\mu}^{*} \psi=\frac{1}{a}\left[\psi(x)-U_{\mu}(x-a \hat{\mu})^{*} \star \psi(x-a \hat{\mu}) \star U_{\mu}(x-a \hat{\mu})\right] .
\end{aligned}
$$

In either case, the fermion action (2.16) is star-gauge invariant.

The second term in the Dirac-Wilson operator (2.17) is the Wilson term, which is introduced to give species doublers a mass of order $\mathcal{O}(1 / a)$. In the original proposal, the coefficient $r$ was taken to be unity, but it can take other values, even negative ones, as far as its magnitude is of order one. 


\subsection{Feynman rules}

Let us proceed to formulate the perturbation theory for the noncommutative QED on the lattice. As in the commutative case we start with expanding the link variable $U_{\mu}(x)$ in terms of the lattice gauge field $A_{\mu}(x)$ as

$$
\begin{aligned}
U_{\mu}(x) & =\exp _{\star}\left\{i a g A_{\mu}(x)\right\} \\
& =1+i g a A_{\mu}(x)-\frac{g^{2} a^{2}}{2} A_{\mu}(x) \star A_{\mu}(x)+\ldots .
\end{aligned}
$$

Note that $U_{\mu}(x)$ is star-unitary if and only if $A_{\mu}(x)$ is real. The Feynman rules are read off from the action (2.16) expressed in terms of the Fourier transformed fields $\tilde{A}_{\mu}(p), \tilde{\psi}(p)$ and $\tilde{\bar{\psi}}(p)$. The fermion propagator is given by

$$
p \rightarrow \quad=\left\{M+\frac{1}{2} r a \hat{p}^{2}-i \gamma \cdot \tilde{p}\right\}^{-1} \equiv Q(p)^{-1}
$$

where we have introduced the notation

$$
\hat{p}_{\mu}=\frac{2}{a} \sin \left(\frac{1}{2} a p_{\mu}\right), \quad \tilde{p}_{\mu}=\frac{1}{a} \sin \left(a p_{\mu}\right) .
$$

That the fermion propagator (2.23) is identical to the one for the commutative lattice QED is because the $\theta$-dependent phase arising from the star-product (2.7) disappears trivially in the quadratic term in the action (Set $p=0$ in (2.7) and consider the anti-symmetry of $\theta_{i j}$ ). The effect of the noncommutativity will show up only in the interaction vertices

in the form of a phase depending on the momenta flowing into them. For the one-photon vertex we find

$$
k, \mu \sim \mathcal{W}_{p}^{(1)}(p, q) V_{\mu}^{(1)}(p+q)
$$

where we have defined

$$
V_{\mu}^{(1)}(p)=i g\left\{\gamma_{\mu} \cos \left(\frac{a}{2} p_{\mu}\right)+i r \sin \left(\frac{a}{2} p_{\mu}\right)\right\} .
$$

The factor $\mathcal{W}^{(1)}(p, q)$ represents a sum of $\theta$-dependent phases, which depends on whether the fermions couple in the fundamental or the adjoint representation

$$
\begin{aligned}
\mathcal{W}_{\text {fund }}^{(1)}(p, q) & =\mathrm{e}^{\frac{i}{2} \theta(\vec{p} \times \vec{q})} \\
\mathcal{W}_{\text {adj }}^{(1)}(p, q) & =\mathrm{e}^{\frac{i}{2} \theta(\vec{p} \times \vec{q})}-\mathrm{e}^{-\frac{i}{2} \theta(\vec{p} \times \vec{q})} .
\end{aligned}
$$

Here we have denoted $\vec{a} \times \vec{b} \equiv-\varepsilon_{j k} a_{j} b_{k}$, where $\varepsilon_{j k}$ is the matrix defined in (2.2). 
For the vertex with two photons we find,

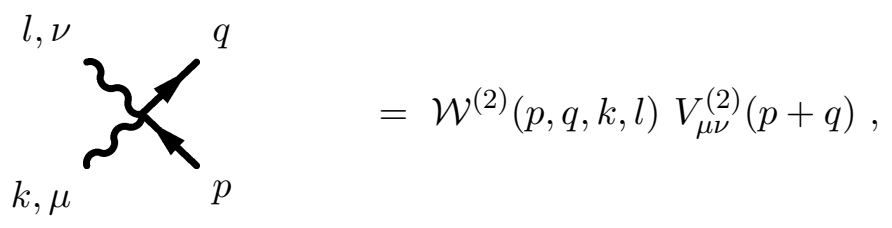

where we defined

$$
V_{\mu \nu}^{(2)}(p)=-a g^{2} \delta_{\mu \nu}\left\{r \cos \left(\frac{a}{2} p_{\mu}\right)+i \gamma_{\mu} \sin \left(\frac{a}{2} p_{\mu}\right)\right\}
$$

The factor $\mathcal{W}^{(2)}(p, q, k, l)$ is now given by

$$
\begin{aligned}
\mathcal{W}_{\text {fund }}^{(2)}(p, q, k, l) & =\mathrm{e}^{\frac{i}{2} \theta(\vec{p} \times \vec{q}+\vec{k} \times \vec{l})} \\
\mathcal{W}_{\text {adj }}^{(2)}(p, q, k, l) & =\mathrm{e}^{\frac{i}{2} \theta(\vec{p} \times \vec{q}+\vec{k} \times \vec{l})}+\mathrm{e}^{\frac{i}{2} \theta(-\vec{p} \times \vec{q}+\vec{k} \times \vec{l})}-\mathrm{e}^{\frac{i}{2} \theta(\vec{p} \times \vec{l}+\vec{k} \times \vec{q})}-\mathrm{e}^{\frac{i}{2} \theta(\vec{p} \times \vec{k}+\vec{l} \times \vec{q})} .
\end{aligned}
$$

Above we assumed that the photon momenta are entering into the vertex.

In addition, for each vertex there is a Kronecker delta momentum conservation

$$
a^{d} L^{d-1} T \delta_{p+k+\cdots, q}
$$

together with a summation over an internal momentum for each loop $\frac{1}{a^{d} L^{d-1} T} \sum_{p}$. Finally, each fermion loop will carry a minus sign. Vertices with more than two photon lines can be obtained in a similar way.

Together with the fermion propagator (2.23), the Feynman rules of noncommutative lattice QED also requires the photon and ghost propagators as well as the photon-ghost and photon self-interaction vertices. In the case of the propagators, because the bilinear terms in the action are independent of the noncommutativity parameter, they are identical to the one for ordinary QED [29]. For the interaction vertices, as it is also the case in the continuum [30], they can be read off from the ones for nonabelian commutative gauge theories given in [29] by simply replacing the structure constants of the gauge group by the appropriate noncommutative phases. In the calculation of the effective action, however, we will need only the fermion-photon vertices.

\subsection{Perturbative evaluation of the effective action}

The effective action for the gauge field is defined in terms of the fermion determinant as

$$
\Gamma[U]_{\mathrm{eff}}=-\log \left[\frac{\operatorname{det}\left(D_{\mathrm{w}}-M\right)}{\operatorname{det}\left(D_{\mathrm{w}, 0}-M\right)}\right]
$$

where $D_{\mathrm{w}, 0}$ is the Dirac-Wilson operator (2.17) evaluated for the trivial gauge configuration $U_{\mu}^{(0)}(x)=1$. Expanding $U_{\mu}(x)$ with respect to $A_{\mu}(x)$ as in $(2.22)$, the effective action $\Gamma[A]_{\mathrm{eff}}$ can be written in momentum space as

$$
\begin{aligned}
\Gamma[A]_{\mathrm{eff}} & =\frac{1}{2} \frac{1}{a^{d} L^{d-1} T} \sum_{p} \Pi_{\mu \nu}(p) A_{\mu}(p) A_{\nu}(-p) \\
& +\frac{1}{3} \frac{1}{\left(a^{d} L^{d-1} T\right)^{2}} \sum_{p, q} \Pi_{\mu \nu \sigma}(p, q) A_{\mu}(p) A_{\nu}(q) A_{\sigma}(-p-q)+\ldots
\end{aligned}
$$


The kernels $\Pi_{\mu \nu}(p), \Pi_{\mu \nu \sigma}(p, q)$ can be computed using the diagrammatic expansion ${ }^{2}$.

$$
-\Pi_{\mu \nu}(p)=\sim 2+\frac{1}{2}\left[\begin{array}{r}
2 \\
-\Pi_{\mu \nu \sigma}(p, q)=
\end{array}\right.
$$

In fact, diagrams containing vertices with three or more photons are irrelevant in the continuum limit since they are weighted with higher powers of the lattice spacing $a[29,31]$. Therefore we can omit the last diagram in (2.35). Applying the Feynman rules, we thus obtain the following expression.

$$
\begin{aligned}
\Pi_{\mu \nu}(p)= & \frac{1}{a^{d} L^{d-1} T} \sum_{q} \operatorname{tr}\left[V_{\mu}^{(1)}(2 q+p) Q(q+p)^{-1} V_{\nu}^{(1)}(2 q+p) Q(q)^{-1}\right] \\
& \times \mathcal{W}^{(1)}(q, q+p) \mathcal{W}^{(1)}(q+p, q) \\
& +\frac{1}{a^{d} L^{d-1} T} \sum_{q} \operatorname{tr}\left[V_{\mu \nu}^{(2)}(2 q) Q(q)^{-1}\right] \mathcal{W}^{(2)}(q, q, p,-p) \\
\Pi_{\mu \nu \sigma}\left(p_{1}, p_{2}\right)= & \frac{1}{a^{d} L^{d-1} T} \sum_{q} \operatorname{tr}\left[V_{\mu}^{(1)}\left(2 q+p_{1}\right) Q\left(q+p_{1}\right)^{-1}\right. \\
& \left.\times V_{\nu}^{(1)}\left(2 q+2 p_{1}+p_{2}\right) Q\left(q+p_{1}+p_{2}\right)^{-1} V_{\sigma}^{(1)}\left(2 q+p_{1}+p_{2}\right) Q(q)^{-1}\right] \\
& \times \mathcal{W}^{(1)}\left(q, q+p_{1}\right) \mathcal{W}^{(1)}\left(q+p_{1}, q+p_{1}+p_{2}\right) \\
& \times \mathcal{W}^{(1)}\left(q+p_{1}+p_{2}, q\right) \\
& +\frac{1}{2 a^{d} L^{d-1} T} \sum_{q}\left\{\operatorname{tr}\left[V_{\mu}^{(1)}\left(2 q+p_{1}\right) Q\left(q+p_{1}\right)^{-1} V_{\nu \sigma}^{(2)}\left(2 q+p_{1}\right) Q(q)^{-1}\right]\right. \\
& \times \mathcal{W}^{(1)}\left(q, q+p_{1}\right) \mathcal{W}^{(2)}\left(q+p_{1}, q, p_{2},-p_{1}-p_{2}\right) \\
& +\operatorname{cyclic} \operatorname{permutations}\}
\end{aligned}
$$

where by "cyclic permutations" we indicate the contributions of the other two diagrams obtained from the second one in Eq. (2.35) by performing cyclic permutations on the labels of the external legs. The expressions for the commutative case can be obtained simply by omitting the factors $\mathcal{W}^{(1)}, \mathcal{W}^{(2)}$ in the above equations.

\section{The parity anomaly in $3 \mathrm{D}$ noncommutative QED}

From now on we will consider the three-dimensional case (i.e. $d \equiv 2 m+1=3$ ) and study the parity anomaly in noncommutative QED on the lattice. Parity anomaly has been studied intensively in commutative gauge theories, both in the continuum $[32,33]$ and on the lattice [21]. It has a wide application in condensed matter physics [34] including the

\footnotetext{
${ }^{2}$ The combinatorial factors in front of the diagrams take into account the overall factors of $\frac{1}{2}$ and $\frac{1}{3}$ multiplying the corresponding terms in the effective action.
} 
quantum Hall effect [35]. We will first briefly review the known results in the commutative case.

\subsection{A brief review of the commutative case}

In three-dimensional massless QED there is a conflict between parity symmetry and gauge invariance at the quantum level. As pointed out in [36] and elaborated in [32], a parity invariant regularization of the fermion determinant leads to non-invariance of the one-loop effective action under large gauge transformations due to the spectral flow of the eigenvalues of the Dirac operator, a phenomenon similar to the one behind Witten's global anomaly [37]. On the other hand, a gauge invariant regularization of the theory, like Pauli-Villars, induces a Chern-Simons action at one loop that breaks parity invariance, with precisely the coefficient required to compensate the variation of the massless fermion determinant under large gauge transformations.

Despite any similarities, parity anomaly in three-dimensional QED is different from ordinary anomalies in that the coefficient of the anomaly depends on the regularization scheme. This peculiar aspect of parity anomaly has been clarified by Coste and Lüscher [21] by using the lattice regularization, which provides the most rigid way to calculate the anomaly while preserving gauge invariance. Here we summarize the main results of Ref. [21]. First, when $M \rightarrow 0$, one obtains in the continuum limit

$$
\lim _{M \rightarrow 0} \Pi_{\mu \nu}(p)=\frac{1}{2 \pi}\left(n+\frac{1}{2}\right) \epsilon_{\mu \nu \sigma} p_{\sigma}+\frac{1}{16|p|}\left(p^{2} \delta_{\mu \nu}-p_{\mu} p_{\nu}\right) .
$$

The term proportional to the Levi-Civita tensor is parity odd, and hence signals the parity anomaly. (Note that the continuum action for a massless Dirac fermion in three dimensions is invariant under parity transformation.) The coefficient of the parity anomaly includes a parameter $n$, which can take any integer value depending on the lattice action chosen, i.e. on the details of the ultraviolet regularization. The essential point, however, is that this regularization ambiguity does not affect the existence of the parity anomaly itself, since this is always nonzero for any $n \in \mathbb{Z}$. For the standard Wilson fermion, one obtains $n=0,-1$, depending on whether the sign of the Wilson term is positive or negative. In this case the parity anomaly arises because the Wilson term breaks parity on the lattice, and this breaking persists in the continuum limit. On the other hand, if one uses the GinspargWilson fermion, whose action is invariant under the generalized parity transformation, the parity anomaly (3.1) arises from the measure [24], thus realizing Fujikawa's philosophy for anomalies at a fully regularized level.

In the infinite mass limit, on the other hand, the result for the vacuum polarization is given by

$$
\begin{aligned}
\lim _{M \rightarrow+\infty} \Pi_{\mu \nu}(p) & =\frac{1}{2 \pi} n \epsilon_{\mu \nu \sigma} p_{\sigma} \\
\lim _{M \rightarrow-\infty} \Pi_{\mu \nu}(p) & =\frac{1}{2 \pi}(n+1) \epsilon_{\mu \nu \sigma} p_{\sigma},
\end{aligned}
$$

where the integer $n$ is the same parameter as the one introduced in (3.1) for the same lattice action. Thus in general the fermion does not decouple completely in the infinite 
mass limit but it leaves behind a certain local term as a remnant. In fact the freedom of the integer parameter $n$ in both (3.1) and (3.2) is closely related to the fact that the remnant (3.2) depends on the sign \pm of the limit $M \rightarrow \pm \infty$. By choosing different lattice action, one essentially introduces different numbers of heavy fermions which have masses of order $\mathcal{O}(1 / a)$. The sign of the masses can be assigned as one wishes, and this results in the arbitrariness represented by $n$.

\subsection{The noncommutative case}

Before we present our results on the noncommutative case, let us remark on what we mean by "parity" when we discuss parity anomaly in noncommutative QED. Conventional parity refers to a reflection in one spatial direction. In the Euclidean formulation in three dimensions, one can combine the conventional parity transformation with the 180 degrees rotation in the remaining two directions, to arrive at the transformation

$$
\begin{aligned}
\psi(x) & \mapsto \psi(-x) \\
\bar{\psi}(x) & \mapsto-\bar{\psi}(-x) \\
A_{\mu}(x) & \mapsto-A_{\mu}(-x),
\end{aligned}
$$

which leaves the massless Dirac action in the continuum invariant. In the noncommutative case, the introduction of the noncommutativity matrix $\theta_{\mu \nu}$ breaks parity in the conventional sense, but it preserves the invariance under (3.3). It is this invariance of the massless Dirac action that we refer to when we say 'parity anomaly' in noncommutative QED.

Our next task is to compute the effective action (2.32) as discussed in Section 2.3 and to see how the results of Coste and Lüscher [21] are modified by noncommutative geometry. We analyze separately the cases of fermions in the fundamental and adjoint representation. We will use a representation of the three-dimensional Dirac matrices satisfying

$$
\gamma_{\mu} \gamma_{\nu}=\delta_{\mu \nu}+i \epsilon_{\mu \nu \sigma} \gamma_{\sigma}
$$

where the matrices are taken to be Hermitian $\gamma_{\mu}^{\dagger}=\gamma_{\mu}$. Hereafter we will fix the sign of $M$ by demanding $M \geq 0$.

\subsubsection{Fundamental fermions}

We begin with the coefficient $\Pi_{\mu \nu}(p)$ of the bilinear term in the effective action $(2.33)$ for the gauge field. For fundamental fermions the noncommutative phases in Eq. (2.36) cancel out. The resulting expression is exactly the same as in the commutative case and in particular the result does not depend on how we take the limits $L \rightarrow \infty, T \rightarrow \infty$ and $a \rightarrow 0$, as far as the physical extent of the space-time $(\ell=a L$ and $\tau=a T)$ goes to infinity. For instance we may take the large volume limit $L \rightarrow \infty$ and $T \rightarrow \infty$ at fixed lattice spacing $a$ and then take the continuum limit $a \rightarrow 0$. Then the rest of the calculation proceeds exactly as in Ref. [21]. Let us introduce the symbol

$$
T_{k}(p) f(p)=\left.\sum_{n=0}^{k} \frac{1}{n !} \frac{\partial^{n}}{\partial t^{n}} f(t p)\right|_{t=0}
$$


which represents a Taylor subtraction at zero momentum. Thus, in the infinite volume limit, $\Pi_{\mu \nu}(p)_{\text {fund }}$ can be rewritten as

$$
\Pi_{\mu \nu}(p)_{\text {fund }}=g^{2} \int_{\mathcal{B}} \frac{d^{3} q}{(2 \pi)^{3}}\left[1-T_{0}(p)\right] \operatorname{tr}\left[Q\left(q-\frac{p}{2}\right)^{-1} \partial_{\mu} Q(q) Q\left(q+\frac{p}{2}\right)^{-1} \partial_{\nu} Q(q)\right],
$$

where the large volume limit $L \rightarrow \infty$ and $T \rightarrow \infty$ has been taken and consequently the momentum sum has been replaced by the integral in the Brillouin zone $\mathcal{B}=\left\{q_{\mu} \in\right.$ $\left.\mathbb{R}^{3} \mid-(\pi / a) \leq q_{\mu} \leq(\pi / a)\right\}$. The subtraction of the zero external momentum contribution comes from the tadpole diagram in the first line of Eq. (2.34). By using the identity $\left[1-T_{0}(p)\right] f(p)=\left[1-T_{1}(p)\right] f(p)+p_{\mu} \partial_{\mu} f(0)$ we can write, after some algebra,

$$
\begin{aligned}
\Pi_{\mu \nu}(p)_{\text {fund }} & =g^{2} a_{0} \epsilon_{\mu \nu \sigma} p_{\sigma} \\
& +g^{2} \int_{\mathcal{B}} \frac{d^{3} q}{(2 \pi)^{3}}\left[1-T_{1}(p)\right] \operatorname{tr}\left[Q\left(q-\frac{p}{2}\right)^{-1} \partial_{\mu} Q(q) Q\left(q+\frac{p}{2}\right)^{-1} \partial_{\nu} Q(q)\right],
\end{aligned}
$$

and

$$
a_{0}=\frac{1}{48 \pi^{3}} \int_{\mathcal{B}} d^{3} q \epsilon_{\mu \nu \sigma} \operatorname{tr}\left[Q(q)^{-1} \partial_{\mu} Q(q) Q(q)^{-1} \partial_{\nu} Q(q) Q(q)^{-1} \partial_{\sigma} Q(q)\right]
$$

As shown in [21], $a_{0}=\frac{1}{2 \pi} n$ is a topological number, where the integer $n$ depends on the parameter $r$, but not on the lattice spacing.

Because of the subtraction at zero momentum, the integral in the second term on the right hand side of (3.7) has negative degree so, according to Reisz theorem [38], its continuum limit is given by the integral over momentum space of the limit of the integrand when $a \rightarrow 0$. On symmetry grounds, the two-point function in the continuum has the form

$$
\Pi_{\mu \nu}(p)=A(p) \epsilon_{\mu \nu \sigma} p_{\sigma}+B(p)\left(p^{2} \delta_{\mu \nu}-p_{\mu} p_{\nu}\right)+C(p) \frac{\theta(p)_{\mu} \theta(p)_{\nu}}{\theta(p)^{2}},
$$

where $\theta(p)_{\mu} \equiv \theta_{\mu \nu} p_{\nu}$. From the previous expressions, we find for the case of fermions in the fundamental representation

$$
\begin{aligned}
& A(p)_{\text {fund }}=g^{2} a_{0}+\frac{g^{2}}{4 \pi} \int_{0}^{1} d x\left\{1-M\left[M^{2}+x(1-x) p^{2}\right]^{-\frac{1}{2}}\right\} \\
& B(p)_{\text {fund }}=\frac{g^{2}}{2 \pi} \int_{0}^{1} d x x(1-x)\left[M^{2}+x(1-x) p^{2}\right]^{-\frac{1}{2}} \\
& C(p)_{\text {fund }}=0
\end{aligned}
$$

In the limits $M \rightarrow 0, M \rightarrow \infty$, we obtain results identical to the commutative case (3.1), (3.2).

Let us proceed to compute the coefficient $\Pi_{\mu \nu \sigma}(p)$ of the trilinear term in the effective action (2.33) for the gauge field. Two types of diagrams contribute to this term. In both cases it is easy to check that the corresponding noncommutative phases are identical and factor out of the sum over the loop momentum. The sum multiplying the global 
noncommutative phase is independent of $\theta$, and it can be evaluated in the same limit as before. Thus we arrive at the expression

$$
\begin{aligned}
\Pi_{\mu \nu \sigma}\left(p_{1}, p_{2}\right)_{\text {fund }}= & e^{\frac{i}{2} \theta\left(\vec{p}_{1} \times \vec{p}_{2}\right)} \int_{\mathcal{B}} \frac{d^{3} q}{(2 \pi)^{3}} \operatorname{tr}\left[V_{\mu}^{(1)}\left(2 q+p_{1}\right) Q\left(q+p_{1}\right)^{-1}\right. \\
& \left.\times V_{\nu}^{(1)}\left(2 q+2 p_{1}+p_{2}\right) Q\left(q+p_{1}+p_{2}\right)^{-1} V_{\sigma}^{(1)}\left(2 q+p_{1}+p_{2}\right) Q(q)^{-1}\right] \\
+ & \frac{1}{2} e^{\frac{i}{2} \theta\left(\vec{p}_{1} \times \vec{p}_{2}\right)}\left\{\int_{\mathcal{B}} \frac{d^{3} q}{(2 \pi)^{3}} \operatorname{tr}\left[V_{\mu}^{(1)}(2 q) Q\left(q-\frac{p_{1}}{2}\right)^{-1} V_{\nu \sigma}^{(2)}(2 q) Q\left(q+\frac{p_{1}}{2}\right)^{-1}\right]\right. \\
& \left.+\left[\text { cyclic permutations: }\left(\mu, p_{1}\right) \rightarrow\left(\nu, p_{2}\right) \rightarrow\left(\sigma,-p_{1}-p_{2}\right)\right]\right\},
\end{aligned}
$$

where we have taken the large volume limit $L \rightarrow \infty$ and $T \rightarrow \infty$, but the continuum limit is yet to be taken. In general, the three-point function can be written as

$$
\Pi_{\mu \nu \sigma}\left(p_{1}, p_{2}\right)_{\text {fund }}=\mathrm{e}^{\frac{i}{2} \theta\left(\vec{p}_{1} \times \vec{p}_{2}\right)} \mathcal{A}\left(p_{1}, p_{2}\right)_{\text {fund }} \epsilon_{\mu \nu \sigma}+\ldots,
$$

where "..." stands for terms proportional to rank-three tensors constructed in terms of the incoming momenta. By looking at the low momentum expansion we find that

$$
\mathcal{A}\left(p_{1}, p_{2}\right)_{\text {fund }}=-g^{3} a_{0}+\mathcal{O}\left(p^{2}\right),
$$

where $a_{0}$ is given again by Eq. (3.8). The remaining momentum-dependent contribution can be obtained by evaluating

$$
\begin{gathered}
\int_{\mathcal{B}} \frac{d^{3} q}{(2 \pi)^{3}}\left[1-T_{0}\left(p_{1}, p_{2}\right)\right] \operatorname{tr}\left[V_{\mu}^{(1)}\left(2 q+p_{1}\right) Q\left(q+p_{1}\right)^{-1} V_{\nu}^{(1)}\left(2 q+2 p_{1}+p_{2}\right)\right. \\
\left.\times Q\left(q+p_{1}+p_{2}\right)^{-1} V_{\sigma}^{(1)}\left(2 q+p_{1}+p_{2}\right) Q(q)^{-1}\right]
\end{gathered}
$$

and keeping the terms proportional to $\epsilon_{\mu \nu \sigma}$. Here we have denoted $T_{0}(p, q) f(p, q)=f(0,0)$. Note also that the second group of terms in (3.11) does not contribute to $\mathcal{A}\left(p_{1}, p_{2}\right)_{\text {fund }}$, since they are symmetric in two of the indices.

In order to retrieve the continuum limit of (3.14) we need to check that the relevant terms of the integral on the right-hand side converges to the continuum Feynman integral. By writing the integrand as $V\left(q, p_{1}, p_{2}, M, a\right) / C\left(q, p_{1}, p_{2}, M, a\right)$ we find that $\operatorname{deg} V \leq 8$, whereas $\operatorname{deg} C=12$, so the degree of divergence of the integrand is $3+\operatorname{deg} V-\operatorname{deg} C \leq-1$, and the contribution of the integral in the continuum limit is given by the integral over the loop momentum of the $a \rightarrow 0$ limit of the integrand. A careful evaluation of the resulting integral using Feynman parameters shows that (see Appendix B for the details)

$$
\begin{aligned}
\lim _{a \rightarrow 0} \mathcal{A}\left(p_{1}, p_{2}\right)_{\text {fund }} & =-g^{3} a_{0}-\frac{g^{3}}{2 \pi} \int_{0}^{1} d x_{1} \int_{0}^{1-x_{1}} d x_{2}\left[1-M\left(M^{2}+\Delta\right)^{-\frac{1}{2}}\right] \\
& -\frac{g^{3}}{4 \pi} M p_{1}^{2} \int_{0}^{1} d x_{1} \int_{0}^{1-x_{1}} d x_{2} \frac{\left(x_{1}+x_{2}\right)\left(1-x_{1}-x_{2}\right)}{\left(M^{2}+\Delta\right)^{\frac{3}{2}}} \\
& -\frac{g^{3}}{4 \pi} M p_{2}^{2} \int_{0}^{1} d x_{1} \int_{0}^{1-x_{1}} d x_{2} \frac{x_{2}\left(1-x_{2}\right)}{\left(M^{2}+\Delta\right)^{\frac{3}{2}}} \\
& -\frac{g^{3}}{8 \pi} M p_{1} \cdot p_{2} \int_{0}^{1} d x_{1} \int_{0}^{1-x_{1}} d x_{2} \frac{x_{1}+2 x_{2}\left(1-x_{1}-x_{2}\right)}{\left(M^{2}+\Delta\right)^{\frac{3}{2}}},
\end{aligned}
$$


where for shorthand we have written

$$
\Delta \equiv\left(x_{1}+x_{2}\right)\left(1-x_{1}-x_{2}\right) p_{1}^{2}+x_{2}\left(1-x_{2}\right) p_{2}^{2}+2 x_{2}\left(1-x_{1}-x_{2}\right) p_{1} \cdot p_{2} .
$$

From these results we see that the cubic term in the noncommutative Chern-Simons action emerges in the limits $M \rightarrow 0$ and $M \rightarrow \infty$ with coefficients consistent with the quadratic term calculated above. This is expected from the star-gauge invariance, which is manifestly preserved in the lattice regularization.

It is straightforward to calculate now the effective action $\Gamma[A]_{\mathrm{eff}}^{(\mathrm{fund})}$ in position space for massless fermions. By using Eqs. (3.10) and (3.15) in the limit $M \rightarrow 0$ we find for the parity violating part

$$
\begin{aligned}
\Gamma[A]_{\mathrm{eff}}^{(\text {fund })} & =\frac{g^{2}}{4 \pi}\left(n+\frac{1}{2}\right) \int \frac{d^{3} p}{(2 \pi)^{3}} \epsilon_{\mu \nu \sigma} \tilde{A}_{\mu}(p) \tilde{A}_{\nu}(p) p_{\sigma} \\
& -\frac{g^{3}}{6 \pi}\left(n+\frac{1}{2}\right) \int \frac{d^{3} p_{1}}{(2 \pi)^{3}} \int \frac{d^{3} p_{1}}{(2 \pi)^{3}} \epsilon_{\mu \nu \sigma} \tilde{A}_{\mu}\left(p_{1}\right) \tilde{A}_{\nu}\left(p_{2}\right) \tilde{A}_{\sigma}\left(-p_{1}-p_{2}\right) \mathrm{e}^{\frac{i}{2} \theta\left(\vec{p}_{1} \times \vec{p}_{2}\right)} .
\end{aligned}
$$

Performing the inverse Fourier transform on $\tilde{A}_{\mu}(p)$ and defining $\mathcal{A}_{\mu}(x)=i g A_{\mu}(x)$ we finally arrive at

$$
\Gamma[\mathcal{A}]_{\mathrm{eff}}^{\text {(fund) }}=\frac{i}{4 \pi}\left(n+\frac{1}{2}\right) \int d^{3} x \epsilon_{\mu \nu \sigma}\left(\mathcal{A}_{\mu} \partial_{\nu} \mathcal{A}_{\sigma}+\frac{2}{3} \mathcal{A}_{\mu} \star \mathcal{A}_{\nu} \star \mathcal{A}_{\sigma}\right)
$$

In particular, since the NCCS term is not invariant under the parity transformation (3.3), we have a parity anomaly as in the commutative case.

It is important to notice that in the $\theta \rightarrow 0$ limit we retrieve the results obtained in Ref. [21] for ordinary (commutative) QED. The fact that the commutative limit turned out to be smooth in the present case is due to the cancellation of the noncommutative phases involving loop momenta, which would otherwise cause the UV/IR mixing. Such a cancellation can be understood in a transparent way [22] if one uses the so-called double-line notation known from large- $N$ gauge theory. Feynman rules should be re-derived accordingly and in particular each of the interaction vertices will have a single noncommutative phase factor instead of a sum of phases. Usefulness of the double-line notation in noncommutative field theories can be understood if one recalls that the algebraic property of the star-product is the same as that of matrix product. In the double-line notation, "planar diagrams" can be defined as diagrams which can be drawn on a plane without any crossings of lines. In fact for any planar diagrams the noncommutative phase factors out of the momentum integration, leaving a global phase depending only on external momenta [39, 40, 41]. For fermions in the fundamental representation, the interaction with the gauge field occurs only on one side of the fermion propagator (represented as a double-line), and therefore all the diagrams that appear in the calculation of the effective action are actually planar.

\subsubsection{Adjoint fermions}

Contrary to the case studied above, the effective action for noncommutative gauge theories with fermions in the adjoint representation does not have a smooth commutative limit. 
Note that the original theory in the $\theta_{\mu \nu} \rightarrow 0$ limit is just a free massless fermion and a free photon, which is parity invariant. However, if we take the continuum limit for finite $\theta$ we will see that the adjoint fermions induce the NCCS action, which reduces to an ordinary (commutative) U(1) Chern-Simons term in the $\theta_{\mu \nu} \rightarrow 0$ limit. Using a PauliVillars fermion of mass $M_{\text {reg }}$ as a regulator in the continuum [23] this is the result of the fact that the two limits $\theta_{\mu \nu} \rightarrow 0$ and $M_{\text {reg }} \rightarrow \infty$ do not commute with each other, a phenomenon characteristic of noncommutative quantum field theories both at zero [22] and finite temperature [44]. This comes about because of the existence of nonplanar diagrams in which the UV divergences are regulated at the scale ${ }^{3} \theta|\vec{p}|$.

Plugging in the factors $\mathcal{W}^{(1)}, \mathcal{W}^{(2)}$ for the adjoint fermions $(2.27),(2.30)$, the expression (2.36) for the two-point function becomes

$$
\begin{aligned}
& \Pi_{\mu \nu}(p)_{\text {adj }}=2 \Pi_{\mu \nu}(p)_{\text {fund }} \\
& -\frac{2}{a^{3} L^{2} T} \sum_{q} \operatorname{tr}\left[V_{\mu}^{(1)}(2 q+p) Q(q+p)^{-1} V_{\nu}^{(1)}(2 q+p) Q(q)^{-1}\right] \cos [\theta(\vec{p} \times \vec{q})] \\
& -\frac{2}{a^{3} L^{2} T} \sum_{q} \operatorname{tr}\left[V_{\mu \nu}^{(2)}(2 q) Q(q)^{-1}\right] \cos [\theta(\vec{p} \times \vec{q})],
\end{aligned}
$$

where $\Pi_{\mu \nu}(p)_{\text {fund }}$ is the two-point function for fermions in the fundamental representation calculated above. In the language of the double-line notation (See the end of Sec. 3.2.1), the first term represents the contribution from the planar diagram and the other two terms correspond to the nonplanar contributions. Interestingly the third term, which is the non-planar contribution from the tadpole diagram, exactly vanishes in the continuum limit. Therefore the effect of the tadpole diagram is just to subtract the zero-momentum contribution from the planar terms, which has the effect of making the amplitude finite in the continuum limit, as seen above. Note that the second term of (3.19) is finite by itself, since the noncommutative phase introduces an effective cutoff to the loop momentum at the scale $\theta|\vec{p}|$.

To obtain the continuum limit of the second term we have to take into account that this limit has to be taken at the same time with the large volume limit in such a way that $\theta \propto a^{2} L$ is kept fixed. A long but straightforward calculation shows that the resulting integrals can be written in terms of modified Bessel functions of the second kind. The resulting two-point function has the form given in Eq. (3.9) now with (see Appendix B for details)

$$
\begin{aligned}
A(p)_{\mathrm{adj}}= & 2 A(p)_{\text {fund }}+\frac{g^{2}}{2 \pi} \sqrt{\frac{2}{\pi}}|\theta(\vec{p})|^{\frac{1}{2}} \int_{0}^{1} d x\left\{M\left[M^{2}+x(1-x) p^{2}\right]^{-\frac{1}{4}}\right. \\
& \left.\times K_{\frac{1}{2}}\left(\theta|\vec{p}|\left[M^{2}+x(1-x) p^{2}\right]^{\frac{1}{2}}\right)\right\} \\
B(p)_{\text {adj }}= & 2 B(p)_{\text {fund }}-\frac{g^{2}}{\pi} \sqrt{\frac{2}{\pi}}|\theta(\vec{p})|^{\frac{1}{2}} \int_{0}^{1} d x\left\{x(1-x)\left[M^{2}+x(1-x) p^{2}\right]^{-\frac{1}{4}}\right.
\end{aligned}
$$

\footnotetext{
${ }^{3}$ The role of nonplanar amplitudes in the calculation of the chiral anomaly has attracted some attention recently (see, for example, [42, 43]).
} 


$$
\begin{aligned}
& \left.\times K_{\frac{1}{2}}\left(\theta|\vec{p}|\left[M^{2}+x(1-x) p^{2}\right]^{\frac{1}{2}}\right)\right\}, \\
C(p)_{\mathrm{adj}}= & \frac{g^{2}}{\pi} \sqrt{\frac{2}{\pi}}|\theta(p)|^{\frac{1}{2}} \int_{0}^{1} d x\left\{\left[M^{2}+x(1-x) p^{2}\right]^{\frac{3}{4}}\right. \\
& \left.\times K_{\frac{3}{2}}\left(\theta|\vec{p}|\left[M^{2}+x(1-x) p^{2}\right]^{\frac{1}{2}}\right)\right\} .
\end{aligned}
$$

Using the asymptotic expansion for large arguments of the Bessel functions, $K_{\nu}(z) \sim$ $e^{z} / \sqrt{2 \pi z}$ it is easy to see that the nonplanar terms in the amplitude are exponentially suppressed in the limit of large $M$ (cf. [23]). In the $M \rightarrow 0$ limit, on the other hand, one finds that the nonplanar contributions to the parity violating term vanishes. Thus in both limits we find that the parity violating term in the effective action comes solely from the planar part, and its magnitude is twice the one for fundamental fermions. Later we will offer a physical explanation of this phenomenon.

For finite values of the noncommutative parameter $\theta$ the three-dimensional Euclidean group $\mathrm{SO}(3)$ is broken down to $\mathrm{SO}(2)$, acting as rotations on the noncommutative plane. An important consequence of the smooth commutative limit $(\theta \rightarrow 0)$ of noncommutative QED with fundamental fermions is that the full Euclidean group $\mathrm{SO}(3)$ is restored in that limit. This means that the "generalized parity" anomaly studied here is mapped to the usual parity anomaly of commutative QED. On the other hand, for adjoint fermions the situation is radically different, since the Euclid-breaking term in the effective action, third equation in (3.20), does not disappear in the commutative limit and actually induces divergences when $\theta \rightarrow 0$, while keeping the $\mathrm{SO}(2)$ symmetry unbroken (see the discussion at the end of this subsection). The reason is that in this case, because of UV/IR mixing, the noncommutative theory is not a smooth deformation of (free) commutative QED with neutral fermions.

Let us now turn to the evaluation of the three-point function. After a short manipulation of the noncommutative phases, the three-point function can be written as

$$
\begin{aligned}
\Pi_{\mu \nu \sigma}\left(p_{1}, p_{2}\right)_{\text {adj }} & =\left[\left(\mathrm{e}^{\frac{i}{2} \theta\left(\vec{p}_{1} \times \vec{p}_{2}\right)}-\mathrm{e}^{-\frac{i}{2} \theta\left(\vec{p}_{1} \times \vec{p}_{2}\right)}\right) \mathcal{A}\left(p_{1}, p_{2}\right)_{\text {fund }}+\mathcal{A}\left(p_{1}, p_{2}, \theta\right)_{\mathrm{NP}}\right] \epsilon_{\mu \nu \sigma} \\
& +\ldots
\end{aligned}
$$

where $\mathcal{A}\left(p_{1}, p_{2}\right)_{\text {fund }}$ is the same function appearing in Eq. (3.12) and $\mathcal{A}\left(p_{1}, p_{2}, \theta\right)_{\mathrm{NP}}$ is the nonplanar contribution. As above the ellipsis denotes further terms whose tensor structure depends on the external momenta. The nonplanar function $\mathcal{A}\left(p_{1}, p_{2}\right)_{\mathrm{NP}}$ is calculated by evaluating the sum

$$
\begin{aligned}
& \frac{1}{a^{3} L^{2} T} \sum_{q} \operatorname{tr}\left[V_{\mu}^{(1)}\left(2 q+p_{1}\right) Q\left(q+p_{1}\right)^{-1} V_{\nu}^{(1)}\left(2 q+2 p_{1}+p_{2}\right)\right. \\
& \left.\quad \times Q\left(q+p_{1}+p_{2}\right)^{-1} V_{\sigma}^{(1)}\left(2 q+p_{1}+p_{2}\right) Q(q)^{-1}\right] \mathcal{W}\left(p_{1}, p_{2}, q\right)_{\mathrm{NP}}
\end{aligned}
$$

and keeping the terms proportional to $\epsilon_{\mu \nu \sigma}$. Here the "nonplanar" part of the noncommutative phase $\mathcal{W}\left(p_{1}, p_{2}, q\right)_{\mathrm{NP}}$ is given by

$$
\begin{aligned}
\mathcal{W}\left(p_{1}, p_{2}, q\right)_{\mathrm{NP}} & =\mathrm{e}^{\frac{i}{2} \theta\left(\vec{p}_{1} \times \vec{p}_{2}\right)}\left[\mathrm{e}^{i \theta\left(\vec{q} \times \vec{p}_{2}\right)}-\mathrm{e}^{-i \theta\left(\vec{q} \times \vec{p}_{1}\right)}-\mathrm{e}^{i \theta \vec{q} \times\left(\vec{p}_{1}+\vec{p}_{2}\right)}\right] \\
& -\mathrm{e}^{-\frac{i}{2} \theta\left(\vec{p}_{1} \times \vec{p}_{2}\right)}\left[\mathrm{e}^{-i \theta\left(\vec{q} \times \vec{p}_{2}\right)}-\mathrm{e}^{i \theta\left(\vec{q} \times \vec{p}_{1}\right)}-\mathrm{e}^{-i \theta \vec{q} \times\left(\vec{p}_{1}+\vec{p}_{2}\right)}\right] .
\end{aligned}
$$


$\mathcal{A}\left(p_{1}, p_{2}\right)_{\mathrm{NP}}$ can be computed in the continuum limit $a \rightarrow 0$ at infinite volume with $a^{2} L$ fixed and expressed in terms of the modified Bessel functions of the second kind. In this limit, $\mathcal{A}\left(p_{1}, p_{2}, \theta\right)_{\mathrm{NP}}$ is expressed in terms of sum of integrals of the form

$$
g^{3} M \int \frac{d^{3} q}{(2 \pi)^{3}} \frac{M^{2}+q^{2}+\left(p_{1}+p_{2}\right) \cdot q}{\left[M^{2}+\left(q+p_{1}\right)^{2}\right]\left[M^{2}+\left(q+p_{1}+p_{2}\right)^{2}\right]\left(M^{2}+q^{2}\right)} \mathrm{e}^{i \theta(\vec{w} \times \vec{q})},
$$

where $\vec{w}=\alpha \vec{p}_{1}+\beta \vec{p}_{2}(\alpha, \beta=0, \pm 1)$ is a linear combination of the incoming momenta, that can be read off from Eq. (3.23). These integrals can be computed again in terms of modified Bessel functions of the second kind. As it was the case also for the two-point functions, all the terms contributing to $\mathcal{A}\left(p_{1}, p_{2}, \theta\right)_{\mathrm{NP}}$ vanish both in the limit $M \rightarrow 0$, due to the global factor of $M$ in front of the integral, and as $M \rightarrow \infty$, this time due to the exponential damping of the modified Bessel function for large values of the argument. The final conclusion is that in the continuum limit

$$
\lim _{M \rightarrow 0} \mathcal{A}\left(p_{1}, p_{2}, \theta\right)_{\mathrm{NP}}=\lim _{M \rightarrow \infty} \mathcal{A}\left(p_{1}, p_{2}, \theta\right)_{\mathrm{NP}}=0 .
$$

As a consequence, in these two limits, the coefficient of the Chern-Simons action is only determined by the first term on the right-hand side of (3.21) and its value is consistent with the one calculated above from the two-point function. This, again, follows from the fact that the lattice regularization preserves star-gauge invariance.

We can now study the parity-odd part of the induced effective action in the limit $M \rightarrow$ 0 . From the two- and three-point amplitudes calculated above we find that $\Gamma[\mathcal{A}]_{\mathrm{eff}}^{(\mathrm{adj})}=$ $2 \Gamma[\mathcal{A}]_{\mathrm{eff}}^{\text {(fund) }}$ where $\Gamma[\mathcal{A}]_{\mathrm{eff}}^{\text {(fund) }}$ is given in Eq. (3.18). Note that we have considered that the adjoint fermions are of Dirac type. The minimal form of the anomaly, however, is obtained by imposing the Majorana condition. In this case there is an extra factor of $\frac{1}{2}$ in front of the fermionic determinant and the effective action for Majorana fermions in the adjoint representation agrees with Eq. (3.18) [23].

To conclude, let us try to understand in physical terms the vanishing of the nonplanar contribution to the parity-violating part of the effective action in the limit of zero fermion mass. As mentioned in Section 3.1 the parity anomaly in commutative gauge theories results from the impossibility of finding a parity-invariant UV cutoff which at the same time preserves Lorentz and gauge symmetries. This is clear from the analysis of [32] where the introduction of a parity-invariant UV cutoff $\Lambda$ in the integrals results in the presence of a term $g^{2} \Lambda \eta_{\mu \nu}$ in the two-point function which breaks the Ward identity.

Because of the presence of the noncommutative phases depending on the loop momentum, we can see the nonplanar contribution to the two-point function of massless noncommutative QED as a regularization of the corresponding amplitude in ordinary (commutative) massless QED. In this case the noncommutative momentum $\theta|\vec{p}| \equiv \Lambda^{-1}$ plays the role of an UV cutoff. Moreover, this cutoff preserve the parity invariance of the theory. Therefore one expects that the parity-breaking terms in the amplitudes will vanish. Indeed, using the nonplanar part in (3.20) we find the two-point function of massless QED in this regularization to be

$$
\Pi_{\mu \nu}(p)_{\mathrm{QED}}^{\theta}=\frac{g^{2}}{2 \pi|p|^{\frac{1}{2}}} \sqrt{\frac{2}{\pi}} \Lambda^{-\frac{1}{2}}\left(p^{2} \delta_{\mu \nu}-p_{\mu} p_{\mu}\right) \int_{0}^{1} d x[x(1-x)]^{\frac{3}{4}} K_{\frac{1}{2}}\left(\Lambda^{-1}\left[x(1-x) p^{2}\right]^{\frac{1}{2}}\right)
$$




$$
-\frac{g^{2}}{2 \pi} \sqrt{\frac{2}{\pi}}|p|^{\frac{3}{2}} \Lambda^{-\frac{1}{2}} \frac{\theta(p)_{\mu} \theta(p)_{\nu}}{\theta(p)^{2}} \int_{0}^{1} d x[x(1-x)]^{\frac{3}{4}} K_{\frac{3}{2}}\left(\Lambda^{-1}\left[x(1-x) p^{2}\right]^{\frac{1}{2}}\right),
$$

i.e. the two-point function does not contain parity-breaking terms and satisfies the Ward identity. However, because of the presence of the last term, it breaks Euclidean symmetry. Thus the " $\theta$-regularization" of massless QED provides a regularization scheme in which both parity and gauge symmetries are maintained at the cost of breaking Euclidean (or Lorentzian) invariance. In the limit in which the cutoff is sent to infinity, $\Lambda \rightarrow \infty$, the coefficient of the first term in the right-hand side of (3.26) tends to $g^{2} /(16|p|)$ whereas the second Euclid-breaking term diverges linearly with $\Lambda$. Euclidean invariance can be restored by introducing a Pauli-Villars fermion with mass $M_{\text {reg }}$ which subtracts the divergent part of the two-point function (3.26). However, this procedure results in the breaking of parity invariance and as a consequence a Chern-Simons term is again induced in the limit $M_{\text {reg }} \rightarrow \infty$. This is somewhat reminiscent to the analysis of nonplanar anomalies in noncommutative gauge theories presented in [43].

\section{Noncommutative Chern-Simons theory on the lattice}

In this section our results in the previous sections are used to define a lattice-regularized noncommutative Chern-Simons theory following the proposal made in Ref. [24] in the commutative case. The basic idea is to use the parity breaking part of the effective action induced by the Ginsparg-Wilson fermion. Since masslessness of the Ginsparg-Wilson fermion is guaranteed on the lattice, one obtains the correct noncommutative Chern-Simons action in the continuum limit without fine-tuning.

The Dirac operator $D$ for the Ginsparg-Wilson fermion is characterized by the GinspargWilson relation [45] which, in the form applicable to both even and odd dimensions, is given by $[24]$

$$
D+D^{\dagger}=a D^{\dagger} D
$$

The general solution to Eq. (4.1) can be written as

$$
D=\frac{1}{a}(1-V)
$$

where $V$ is a unitary operator, which should turn into the identity operator in the naive continuum limit. In even dimensions, assuming further the " $\gamma_{5}$-Hermiticity"

$$
D^{\dagger}=\gamma_{5} D \gamma_{5}
$$

one arrives at the original Ginsparg-Wilson relation [45]

$$
D \gamma_{5}+\gamma_{5} D=a D \gamma_{5} D
$$

This relation guarantees that the fermion action including the operator $D$ is invariant under a generalized chiral symmetry [46], which reduces to the ordinary chiral symmetry in the continuum limit. The role of the " $\gamma_{5}$-Hermiticity" is played in odd dimensions by the property

$$
D(U)^{\dagger}=\mathcal{R} D\left(U^{P}\right) \mathcal{R},
$$


where $U^{P}$ is the parity transformed gauge configuration,

$$
U_{\mu}^{P}(x)=U_{\mu}(-x)^{\dagger},
$$

and $\mathcal{R}$ is the space-time reflection operator, $\mathcal{R}: x \mapsto-x$. Combining (4.1) and (4.5) one can show the invariance of the corresponding fermion action under a generalized parity transformation [24]. The measure, however, is not invariant under the same transformation. As a consequence, the fermion determinant is not invariant but transforms as

$$
\operatorname{det} D\left(U^{P}\right)=(\operatorname{det} V)^{*} \operatorname{det} D(U) \text {. }
$$

So far we have discussed general properties of the Ginsparg-Wilson operator, which satisfies (4.1). In fact the unitary operator $V$ has to be chosen appropriately in order to guarantee that the operator $D$ has sensible properties as a Dirac operator such as locality (with exponentially decaying tails) and the absence of species doublers. Such an operator has been derived from the overlap formalism [47], and it is given explicitly by [48]

$$
\begin{aligned}
V & =A_{\mathrm{w}} / \sqrt{A_{\mathrm{w}}^{\dagger} A_{\mathrm{w}}} \\
A_{\mathrm{w}} & =1-a D_{\mathrm{w}}(r=-1),
\end{aligned}
$$

where $D_{\mathrm{w}}(r=-1)$ is the Dirac-Wilson operator, which has the form (2.17) with ordinary covariant derivatives. The noncommutative version of the Ginsparg-Wilson fermion can be obtained by simply using the covariant derivatives (2.19) or (2.21) depending on the representation, instead of the usual ones without star-products. In even dimensions Ginsparg-Wilson fermions played a crucial role in introducing chirality on a discretized noncommutative torus [20]. Recently an analogous construction has been worked out on a fuzzy sphere [49].

For the choice (4.8), $\operatorname{det} V$ in (4.7) is nothing but the phase of $\operatorname{det} A_{\mathrm{w}}$, which is essentially the fermion determinant of the Wilson-Dirac operator with $r=-1$ and $M=1 / a$. Thus one can translate the result obtained for the Wilson fermion in the infinite mass limit into the parity anomaly for the Ginsparg-Wilson fermion. In the commutative case this is how the correct parity anomaly has been reproduced by [50] in the overlap formalism ${ }^{4}$. In the noncommutative case, on the other hand, our results in the previous section with $r=-1$ in the limit $M \rightarrow \infty$ implies that the parity anomaly obtained for Ginsparg-Wilson fermions coincides with the result for Wilson fermions with $r=-1$ in the massless limit $M \rightarrow 0$.

As in the commutative case [24], the parity anomaly for the Ginsparg-Wilson fermion suggests a natural definition of the noncommutative Chern-Simons term on the lattice. Namely we define it as $S_{\mathrm{CS}}$ in

$$
\mathrm{e}^{i S_{\mathrm{CS}}} \stackrel{\operatorname{def}}{=} \frac{\operatorname{det} A_{\mathrm{w}}}{\left|\operatorname{det} A_{\mathrm{w}}\right|}
$$

where $A_{\mathrm{w}}$ is defined by (4.9) with the covariant derivative (2.19) for the fundamental representation. Here we remind the reader that $A_{\mathrm{W}}$ is related to the infinite-mass Dirac

\footnotetext{
${ }^{4}$ See also [51] for an earlier work on the overlap formalism in odd dimensions, where a parity invariant phase choice has been made.
} 
determinant as we discussed in the previous paragraph. According to our calculations, the quantity $S_{\mathrm{CS}}$ indeed becomes the noncommutative Chern-Simons action in the continuum limit. In the continuum, on the other hand, noncommutative Chern-Simons term is known to transform as $[52]^{5}$

$$
S_{\mathrm{CS}} \mapsto S_{\mathrm{CS}}+2 \pi \nu
$$

under a gauge transformation, where $\nu$ is the winding number characterizing this gauge transformation. The gauge invariance requires the coefficient of the noncommutative Chern-Simons action to be quantized. That Eq. (4.10) defines $S_{\mathrm{CS}}$ only up to modulo $2 \pi$ is therefore not a problem for most practical purposes. Note in this regard that the right-hand side of eq. (4.10) is indeed manifestly gauge invariant.

\section{Concluding remarks}

In the present paper we have studied the emergence of parity anomaly on the lattice for three-dimensional noncommutative QED, both with fermions in the fundamental and the adjoint representation. Induced Chern-Simons actions in noncommutative gauge theories have been studied in the continuum in $[23,54]$ using the Pauli-Villars regularization (see also [55] for an incomplete list of references). However, the main advantage of the lattice analysis presented here lies in making explicit the dependence of the coefficient of the induced ChernSimons term on the regularization scheme used. Thus, the results obtained in Ref. [23] corresponds to the cases $n=0,-1$ in our analysis using the lattice regularization. Notice that the quantization of the scheme-dependent term in the effective action is consistent with the star-gauge invariance of the fermion determinant under "large" transformations [52], as required by the fact that the lattice regularization respects star-gauge invariance.

We have also proposed a lattice-regularized Chern-Simons action on a noncommutative torus using Ginsparg-Wilson fermions. As the lattice formulation of noncommutative field theories has been useful to extract their interesting nonperturbative dynamics $[15,16,18]$, we hope that the lattice formulation of noncommutative Chern-Simons action is useful to deepen our understanding of quantum Hall systems.

Finally we would like to emphasize that the lattice noncommutative field theories studied in the present paper can be mapped on to a finite $N$ matrix model. The anomaly calculation in matrix models has recently attracted attention in the context of large- $N$ gauge theory $[56,57]$ and noncommutative geometry $[58,49]$. We expect that the calculation method developed in this paper is useful to study various anomalies in noncommutative geometry. In particular we would like to revisit the gauge anomaly cancellation in chiral gauge theories on a noncommutative torus [20]. We hope that such developments will eventually lead us to a deeper understanding of the stringy nature of the space-time structure.

\footnotetext{
${ }^{5}$ This gauge violation was also concluded in Ref. [53], although it was overlooked in its first version.
} 


\section{Acknowledgments}

We would like to thank L. Alvarez-Gaumé, A. Armoni, J.L.F. Barbón, M. García-Pérez, S. Iso, H. Kawai, Y. Kikukawa, K.E. Kunze and D. Miller for helpful discussions. The work of J.N. is supported in part by Grant-in-Aid for Scientific Research (No. 14740163) from the Ministry of Education, Culture, Sports, Science and Technology. M.A.V.-M. acknowledges partial support from Spanish Science Ministry Grants AEN99-0315 and FPA2002-02037.

\section{A. Functional determinants}

In the following we will provide an alternative calculation of the effective action $\Gamma[A]_{\mathrm{eff}}$ on the lattice by direct evaluation of the fermionic determinant (2.32). From the definition of the Dirac-Wilson operator (2.17) and the expansion of the link field $U_{\mu}(x)$ in terms of the lattice gauge field $A_{\mu}(x)(2.22)$, one can write

$$
D_{\mathrm{w}}=\sum_{k=0}^{\infty} g^{k} D_{\mathrm{w}, k} .
$$

Using this expansion, the effective action can be expressed as

$$
\begin{aligned}
\Gamma[A]_{\mathrm{eff}} & =-\log \left[\frac{\operatorname{det}\left(D_{\mathrm{w}}-M\right)}{\operatorname{det}\left(D_{\mathrm{w}, 0}-M\right)}\right]=-\log \operatorname{det}\left[1+\left(D_{\mathrm{w}, 0}-M\right)^{-1} \sum_{k=1}^{\infty} g^{k} D_{\mathrm{w}, k}\right] \\
& =-\operatorname{Tr} \log \left[1+\sum_{k=1}^{\infty} g^{k}\left(D_{\mathrm{w}, 0}-M\right)^{-1} D_{\mathrm{w}, k}\right],
\end{aligned}
$$

which leads to the following series for $\Gamma[A]_{\mathrm{eff}}$ :

$$
\Gamma[A]_{\mathrm{eff}}=g^{2} \Gamma_{2}[A]+g^{3} \Gamma_{3}[A]+\ldots
$$

with

$$
\begin{aligned}
\Gamma_{2}[A]= & \operatorname{Tr}\left\{\frac{1}{2}\left[\left(D_{\mathrm{w}, 0}-M\right)^{-1} D_{\mathrm{w}, 1}\right]^{2}-\left(D_{\mathrm{w}, 0}-M\right)^{-1} D_{\mathrm{w}, 2}\right\} \\
\Gamma_{3}[A]= & \operatorname{Tr}\left\{-\frac{1}{3}\left[\left(D_{\mathrm{w}, 0}-M\right)^{-1} D_{\mathrm{w}, 1}\right]^{3}+\left[\left(D_{\mathrm{w}, 0}-M\right)^{-1} D_{\mathrm{w}, 1}\left(D_{\mathrm{w}, 0}-M\right)^{-1} D_{\mathrm{w}, 2}\right]\right. \\
& \left.-\left(D_{\mathrm{w}, 0}-M\right)^{-1} D_{\mathrm{w}, 3}\right\} .
\end{aligned}
$$

By comparing with Eqs. (2.34) and (2.35) we can identify each term in the previous equations with the contribution of a particular Feynman diagram.

A quick computation shows that for fermions in the fundamental representation the operator $D_{\mathrm{w}, k}$ appearing in Eq. (A.1) is given by

$$
\begin{aligned}
D_{\mathrm{w}, k}^{(\text {fund })} \psi(x)= & \frac{(i a)^{k}}{2 a k !} \sum_{\mu=1}^{d}\left[\left(r+\gamma_{\mu}\right) A_{\mu}(x)^{\star k} \star \psi(x+a \hat{\mu})\right. \\
& \left.+(-1)^{k}\left(r-\gamma_{\mu}\right) A_{\mu}(x-a \hat{\mu})^{\star k} \star \psi(x-a \hat{\mu})\right],
\end{aligned}
$$


whereas when the fermions are in the adjoint representation the result is

$$
\begin{aligned}
D_{\mathrm{w}, k}^{(\mathrm{adj})} \psi(x)= & \frac{(i a)^{k}}{2 a k !} \sum_{\mu=1}^{d} \sum_{m=0}^{k}(-1)^{m}\left(\begin{array}{c}
k \\
m
\end{array}\right)\left[\left(r+\gamma_{\mu}\right) A_{\mu}(x)^{\star(k-m)} \star \psi(x+a \hat{\mu}) \star A_{\mu}(x)^{\star m}\right. \\
& \left.+\left(r-\gamma_{\mu}\right) A_{\mu}(x-a \hat{\mu})^{\star m} \star \psi(x-a \hat{\mu}) \star A_{\mu}(x-a \hat{\mu})^{\star(k-m)}\right] .
\end{aligned}
$$

In both expressions we have used the notation $\phi(x)^{\star n} \equiv \overbrace{\phi(x) \star \ldots \star \phi(x)}^{n}$.

In order to evaluate each term in Eq. (A.4)-(A.5) it is convenient to work in momentum space. In the following we will detail the calculation for fundamental fermions, leaving the adjoint case for the reader. Using Eq. (2.5) together with

$$
\tilde{A}_{\mu}(p)=a^{3} \sum_{x \in \Lambda_{L, T}} A_{\mu}(x) \mathrm{e}^{-i p \cdot\left(x+\frac{1}{2} a \hat{\mu}\right)}
$$

one can easily find the action of the operators in Eqs. (A.4)-(A.5) on $\tilde{\psi}(p)$. For the free propagator we have

$$
\left(D_{\mathrm{w}, 0}-M\right)^{-1} \tilde{\psi}(p)=-Q(p)^{-1} \tilde{\psi}(p),
$$

whereas the result for $D_{\mathrm{w}, 1}$ can be written as

$$
\begin{aligned}
g D_{1} \tilde{\psi}(p)= & \frac{i g}{a^{3} L^{2} T} \sum_{q \in \mathcal{B}} \sum_{\mu=1}^{3}\left\{\gamma_{\mu} \cos \left[\frac{a}{2}(p+q)_{\mu}\right]+i r \sin \left[\frac{a}{2}(p+q)_{\mu}\right]\right\} \mathrm{e}^{\frac{i}{2} \theta(\vec{p} \times \vec{q})} \\
& \times \tilde{A}_{\mu}(p-q) \tilde{\psi}(q) \\
= & \frac{1}{a^{3} L^{2} T} \sum_{q \in \mathcal{B}} \sum_{\mu=1}^{3} V_{\mu}^{(1)}(p+q) \mathcal{W}_{\text {fund }}^{(1)}(p, q) \tilde{A}_{\mu}(p-q) \tilde{\psi}(q) .
\end{aligned}
$$

Here $V_{\mu}^{(1)}(p)$ and $\mathcal{W}_{\text {fund }}^{(1)}(p, q)$ are defined in Eqs. (2.26) and (2.27) respectively. For $D_{\mathrm{w}, 2}$ we arrive at

$$
\begin{aligned}
g^{2} D_{\mathrm{w}, 2} \tilde{\psi}(p)= & -\frac{a g^{2}}{2\left(a^{3} L^{2} T\right)^{2}} \sum_{q, q^{\prime} \in \mathcal{B}} \sum_{\mu=1}^{3}\left\{r \cos \left[\frac{a}{2}(p+q)_{\mu}\right]+i \gamma_{\mu} \sin \left[\frac{a}{2}(p+q)_{\mu}\right]\right\} \\
& \times \mathrm{e}^{\frac{i}{2} \theta\left[\vec{p} \times \vec{q}+\vec{q}^{\prime} \times\left(\vec{p}-\vec{q}-\vec{q}^{\prime}\right)\right]} \tilde{A}_{\mu}\left(q^{\prime}\right) \tilde{A}_{\mu}\left(p-q-q^{\prime}\right) \tilde{\psi}(q) \\
= & \frac{1}{2\left(a^{3} L^{2} T\right)^{2}} \sum_{q, q^{\prime} \in \mathcal{B}} \sum_{\mu, \nu=1}^{3} V_{\mu \nu}^{(2)}(p+q) \mathcal{W}_{\text {fund }}^{(2)}\left(p, q, q^{\prime}, p-q-q^{\prime}\right) \\
& \times \tilde{A}_{\mu}\left(q^{\prime}\right) \tilde{A}_{\nu}\left(p-q-q^{\prime}\right) \tilde{\psi}(q) .
\end{aligned}
$$

As in the previous case we have introduced the vertex function and the noncommutative phase defined in Eqs. (2.29) and (2.30) respectively. Finally, for $D_{\mathrm{w}, 3}$ the result is

$$
\begin{aligned}
g^{3} D_{\mathrm{w}, 3} \tilde{\psi}(p)= & \frac{a g^{3}}{3 !\left(a^{3} L^{2} T\right)^{3}} \sum_{q, q^{\prime}, q^{\prime \prime} \in \mathcal{B}} \sum_{\mu=1}^{3}\left\{r \sin \left[\frac{a}{2}(p+q)_{\mu}\right]-i \gamma_{\mu} \cos \left[\frac{a}{2}(p+q)_{\mu}\right]\right\} \\
& \times \mathrm{e}^{\frac{i}{2} \theta\left[\vec{q} \times \vec{q}^{\prime}+\vec{q} \times \vec{q}^{\prime \prime}+\vec{q}^{\prime} \times \vec{q}^{\prime \prime}+\vec{p} \times\left(\vec{q}-\vec{q}^{\prime}-\vec{q}^{\prime \prime}\right)\right]} \\
& \times \tilde{A}_{\mu}\left(q^{\prime}\right) \tilde{A}_{\mu}\left(q^{\prime \prime}\right) \tilde{A}_{\mu}\left(p-q-q^{\prime}-q^{\prime \prime}\right) \tilde{\psi}(q) .
\end{aligned}
$$


This term is associated with the three-photon vertex in the diagramatic expansion that, as argued in Section 2.2, is irrelevant in the continuum limit.

We have seen that, when written in momentum space, all operators appearing in Eqs. (A.4)-(A.5) are expressed as finite matrices, $\mathcal{O} \tilde{\psi}(p)=\sum_{q \in \mathcal{B}} \mathcal{O}(p, q) \tilde{\psi}(q)$, whose traces can be easily calculated. Let us begin with $\Gamma_{2}[A]$. The first trace to be computed is

$$
\begin{array}{r}
g^{2} \operatorname{Tr}\left\{\frac{1}{2}\left[\left(D_{\mathrm{w}, 0}-M\right)^{-1} D_{\mathrm{w}, 1}\right]^{2}\right\}=\frac{1}{2\left(a^{3} L^{2} T\right)^{2}} \sum_{\mu, \nu=1}^{3} \sum_{p, q \in \mathcal{B}} \tilde{A}_{\mu}(p) \tilde{A}_{\nu}(-p) \\
\times \operatorname{tr}\left[V_{\mu}^{(1)}(2 q+p) Q(q+p)^{-1} V_{\nu}^{(1)}(2 q+p) Q(q)^{-1}\right]
\end{array}
$$

where "tr" indicates the trace over Dirac indices. In the same way, for the second trace in (A.4) the result is

$$
\begin{aligned}
-g^{2} \operatorname{Tr}\left[\left(D_{\mathrm{w}, 0}-M\right)^{-1} D_{\mathrm{w}, 2}\right]= & \frac{1}{2\left(a^{3} L^{2} T\right)^{2}} \sum_{\mu, \nu=1}^{3} \sum_{p, q \in \mathcal{B}} \tilde{A}_{\mu}(p) \tilde{A}_{\nu}(-p) \\
& \times \operatorname{tr}\left[V_{\mu \nu}^{(2)}(2 q) Q(q)^{-1}\right] .
\end{aligned}
$$

Adding these two terms to get $\Gamma_{2}[A]$ and extracting the kernel $\Pi_{\mu \nu}(p)$ defined in $(2.33)$, we recover Eq. (2.36).

The cubic term $\Gamma_{3}[A]$ in the effective action can be computed along similar lines. The first term in (A.5) gives

$$
\begin{aligned}
-\frac{1}{3} \operatorname{Tr}\{ & {\left.\left[\left(D_{\mathrm{w}, 0}-M\right)^{-1} D_{\mathrm{w}, 1}\right]^{3}\right\}=\frac{1}{3\left(a^{3} L^{2} T\right)^{3}} \sum_{\mu, \nu, \sigma=1}^{3} \sum_{p, q, q^{\prime} \in \mathcal{B}} \tilde{A}_{\mu}(q) \tilde{A}_{\nu}\left(q^{\prime}\right) \tilde{A}_{\sigma}\left(-q-q^{\prime}\right) } \\
& \times \mathrm{e}^{\frac{i}{2} \theta\left(\vec{q} \times \vec{q}^{\prime}\right)} \operatorname{tr}\left[V_{\mu}^{(1)}(2 p+q) Q(p+q)^{-1} V_{\nu}^{(1)}\left(2 p+2 q+q^{\prime}\right) Q\left(p+q+q^{\prime}\right)^{-1}\right. \\
& \left.\times V_{\sigma}^{(1)}\left(2 p+q+q^{\prime}\right) Q(p)^{-1}\right]
\end{aligned}
$$

whereas the second trace renders

$$
\begin{aligned}
\operatorname{Tr} & {\left[\left(D_{\mathrm{w}, 0}-M\right)^{-1} D_{\mathrm{w}, 1}\left(D_{\mathrm{w}, 0}-M\right)^{-1} D_{\mathrm{w}, 2}\right] } \\
& =\frac{1}{2\left(a^{3} L^{2} T\right)^{3}} \sum_{\mu, \nu, \sigma=1}^{3} \sum_{p, q, q^{\prime} \in \mathcal{B}} \tilde{A}_{\mu}(q) \tilde{A}_{\nu}\left(q^{\prime}\right) \tilde{A}_{\sigma}\left(-q-q^{\prime}\right) \\
& \times \mathrm{e}^{\frac{i}{2} \theta\left(\vec{q} \times \vec{q}^{\prime}\right)} \operatorname{tr}\left[V_{\mu}^{(1)}(2 p+q) Q(p+q)^{-1} V_{\nu \sigma}^{(2)}(2 p+q) Q(p+q)^{-1}\right] .
\end{aligned}
$$

The third term $\operatorname{Tr}\left\{\left[D_{\mathrm{w}, 0}-M\right]^{-1} D_{\mathrm{w}, 3}\right\}$ corresponds to the contribution of the tadpole diagram which is irrelevant in the continuum limit. Adding together (A.15) and (A.16), and identifying the kernel $\Pi_{\mu \nu \sigma}$, we recover the result of Eq. (2.37).

In the case of adjoint fermions, the calculation is analogous to the one describe above, the main difference being the noncommutative phases. Again the results of Section 2.3 are recovered. 


\section{B. Evaluation of the Feynman integrals}

In this Appendix we will provide the reader with details of the calculation of some of the Feynman integrals in Sections 3.2.1 and 3.2.2. As explained above, we consider a hermitian representation of the $2 \times 2$ gamma matrices satisfying $\gamma_{\mu} \gamma_{\nu}=\delta_{\mu \nu}+i \epsilon_{\mu \nu \sigma} \gamma_{\sigma}$. This implies the following trace identities:

$$
\begin{aligned}
\operatorname{tr}\left(\gamma_{\mu} \gamma_{\nu} \gamma_{\sigma}\right) & =2 i \epsilon_{\mu \nu \sigma} \\
\operatorname{tr}\left(\gamma_{\mu} \gamma_{\nu} \gamma_{\sigma} \gamma_{\lambda}\right) & =2\left(\delta_{\mu \nu} \delta_{\sigma \lambda}+\delta_{\mu \lambda} \delta_{\nu \sigma}-\delta_{\mu \sigma} \delta_{\nu \lambda}\right) \\
\operatorname{tr}\left(\gamma_{\mu} \gamma_{\nu} \gamma_{\sigma} \gamma_{\lambda} \gamma_{\alpha}\right) & =2 i\left(\delta_{\mu \nu} \epsilon_{\sigma \lambda \alpha}+\delta_{\lambda \alpha} \epsilon_{\mu \nu \sigma}+\delta_{\sigma \lambda} \epsilon_{\mu \nu \alpha}-\delta_{\sigma \alpha} \epsilon_{\mu \nu \lambda}\right) \\
\operatorname{tr}\left(\gamma_{\mu} \gamma_{\nu} \gamma_{\sigma} \gamma_{\lambda} \gamma_{\alpha} \gamma_{\beta}\right) & =2\left(\delta_{\mu \nu} \delta_{\sigma \lambda} \delta_{\alpha \beta}+\delta_{\mu \nu} \delta_{\sigma \beta} \delta_{\lambda \alpha}-\delta_{\mu \nu} \delta_{\sigma \alpha} \delta_{\lambda \beta}-\delta_{\alpha \beta} \delta_{\mu \sigma} \delta_{\nu \lambda}\right. \\
& +\delta_{\alpha \beta} \delta_{\mu \lambda} \delta_{\nu \sigma}-\delta_{\lambda \alpha} \delta_{\mu \sigma} \delta_{\nu \beta}+\delta_{\lambda \alpha} \delta_{\mu \beta} \delta_{\nu \sigma}+\delta_{\lambda \beta} \delta_{\mu \sigma} \delta_{\nu \alpha} \\
& \left.-\delta_{\lambda \beta} \delta_{\mu \alpha} \delta_{\nu \sigma}-\epsilon_{\mu \nu \sigma} \epsilon_{\lambda \alpha \beta}\right)
\end{aligned}
$$

\section{B.1 Fundamental fermions}

As shown in [21], the integrand of the second term in Eq. (3.7) has negative degree so the continuum limit exists and gives rise to the integral

$$
I_{\mu \nu}=-g^{2} \int \frac{d^{3} q}{(2 \pi)^{3}}\left[1-T_{1}(p)\right] \frac{\operatorname{tr}\left\{\gamma_{\mu}[M+i(\not 1+\not p)] \gamma_{\nu}[M+i \not q]\right\}}{\left[M^{2}+(q+p)^{2}\right]\left(M^{2}+q^{2}\right)} .
$$

By using the trace identities (B.1) and writing the denominator as an integral over a Feynman parameter

$$
\frac{1}{\left[M^{2}+(q+p)^{2}\right]\left(M^{2}+q^{2}\right)}=\int_{0}^{1} d x\left[(q+x p)^{2}+M^{2}+x(1-x) p^{2}\right]^{-2},
$$

one arrives at

$$
\begin{aligned}
I_{\mu \nu}= & -2 g^{2} \int_{0}^{1} d x \int \frac{d^{3} q}{(2 \pi)^{3}}\left[1-T_{1}(p)\right]\left\{M \epsilon_{\mu \nu \alpha} p_{\alpha}-2 q_{\mu \nu}+2 x(1-x) p_{\mu} p_{\nu}\right. \\
& \left.+\left[M^{2}+q^{2}-x(1-x) p^{2}\right] \delta_{\mu \nu}\right\}\left[q^{2}+M^{2}+x(1-x) p^{2}\right]^{-2} .
\end{aligned}
$$

The final result (3.10) is readily obtained by computing the momentum integral. Note that, because of the zero-momentum subtraction, the integral in (B.4) is free of divergences.

We now evaluate the function $\mathcal{A}\left(p_{1}, p_{2}\right)_{\text {fund }}$ in (3.12). The relevant integral to calculate is the continuum limit of Eq. (3.14) which can be cast into

$$
\begin{aligned}
I_{\mu \nu \sigma}= & -i g^{3} \int \frac{d^{3} q}{(2 \pi)^{3}}\left[1-T_{0}\left(p_{1}, p_{2}\right)\right] \\
& \times \frac{\operatorname{tr}\left\{\gamma_{\mu}\left[M+i\left(\not q+\not p_{1}\right)\right] \gamma_{\nu}\left[M+i\left(\not q+\not p_{1}+\not p_{2}\right)\right] \gamma_{\sigma}(M+i \not q)\right\}}{\left[M^{2}+\left(q+p_{1}\right)^{2}\right]\left[M^{2}+\left(q+p_{1}+p_{2}\right)^{2}\right]\left(M^{2}+q^{2}\right)} .
\end{aligned}
$$

In order to compute $\mathcal{A}\left(p_{1}, p_{2}\right)$ fund we need to retain only those terms proportional to the Levi-Civita tensor $\epsilon_{\mu \nu \sigma}$. By expanding the trace in the numerator and using Eqs. (B.1) it 
is straightforward to check that only two terms, proportional to $M^{3}$ and $M$, contribute to $\mathcal{A}\left(p_{1}, p_{2}\right)_{\text {fund }}$, namely

$$
\begin{aligned}
\mathcal{A}\left(p_{1}, p_{2}\right)_{\text {fund }}= & 4 M g^{3} \int \frac{d^{3} q}{(2 \pi)^{3}} \int_{0}^{1} d x_{1} \int_{0}^{1-x_{2}} d x_{2}\left[1-T_{0}\left(p_{1}, p_{2}\right)\right] \\
& \times \frac{M^{2}+q^{2}+\left(p_{1}+p_{2}\right) \cdot q}{\left\{\left[q+\left(x_{1}+x_{2}\right) p_{1}+x_{2} p_{2}\right]^{2}+\Delta\right\}^{3}},
\end{aligned}
$$

where we have reduced the denominator of (B.5) by introducing Feynman parameters and $\Delta$ is defined in Eq. (3.16). The integral can be easily evaluated using standard techniques to find the result given in Eq. (3.15).

\section{B.2 Adjoint fermions}

In the case when the fermions are in the adjoint representation, the evaluation of the Feynman integrals is more involved due to the presence of noncommutative phases dependent on the loop momentum. Let us focus first on the two-point function. As explained above, contrary to the planar part where the tadpole diagram introduces the zero momentum subtraction that makes the whole amplitude finite, in the nonplanar sector the tadpole diagram cancels exactly. This can be seen by noticing that, up to a total derivative term that cancels in the continuum limit, the nonplanar contribution of the tadpole diagram in the continuum limit is given by

$$
\begin{aligned}
\left.\Pi_{\mu \nu}(p, \theta)_{\text {adj }}\right|_{\text {tadpole }} & =-4 g^{2} \int \frac{d^{3} q}{(2 \pi)^{3}} \frac{\left(M^{2}+q^{2}\right) \delta_{\mu \nu}-2 q_{\mu} q_{\nu}}{\left(q^{2}+M^{2}\right)^{2}} \cos [\theta(\vec{p} \times \vec{q})] \\
& +4 g^{2} \theta(p)_{\nu} \int \frac{d^{3} q}{(2 \pi)^{3}} \frac{q_{\mu}}{q^{2}+M^{2}} \sin [\theta(\vec{p} \times \vec{q})] .
\end{aligned}
$$

The relevant integrals can be easily solved in terms of modified Bessel functions of the second kind $(\omega \in \mathbb{R})$ :

$$
\begin{aligned}
\int \frac{d^{3} q}{(2 \pi)^{3}} \frac{2 q_{\mu} q_{\nu}-q^{2} \delta_{\mu \nu}}{\left(q^{2}+\omega^{2}\right)^{2}} \mathrm{e}^{i \theta(\vec{p} \times \vec{q})}= & \frac{1}{8 \pi} \sqrt{\frac{2}{\pi}}|\omega|^{\frac{3}{2}}(\theta|\vec{p}|)^{\frac{1}{2}}\left\{K_{\frac{1}{2}}(\theta|\vec{p}||\omega|) \delta_{\mu \nu}\right. \\
& \left.-2 K_{\frac{3}{2}}(\theta|\vec{p}||\omega|) \frac{\theta(p)_{\mu} \theta(p)_{\nu}}{\theta(p)^{2}}\right\}, \\
\int \frac{d^{3} q}{(2 \pi)^{3}} \frac{\mathrm{e}^{i \theta(\vec{p} \times \vec{q})}}{\left(q^{2}+\omega^{2}\right)^{2}} & =\frac{1}{8 \pi} \sqrt{\frac{2}{\pi}}|\omega|^{-\frac{1}{2}}(\theta|\vec{p}|)^{\frac{1}{2}} K_{\frac{1}{2}}(\theta|\vec{p}||\omega|), \\
\int \frac{d^{3} q}{(2 \pi)^{3}} \frac{q_{\mu}}{q^{2}+\omega^{2}} \mathrm{e}^{i \theta(\vec{p} \times \vec{q})} & =\frac{i}{4 \pi} \frac{\theta(p)_{\mu}}{\theta(p)^{2}} \sqrt{\frac{2}{\pi}}(\theta|\vec{p}|)^{\frac{1}{2}}|\omega|^{\frac{3}{2}} K_{\frac{3}{2}}(\theta|\vec{p}||\omega|) .
\end{aligned}
$$

Substituting these expressions into (B.7) one finds a cancellation between the different terms.

As for the nonplanar part of the two-point function coming from the first diagram in Eq. (2.34), the relevant integral to evaluate is

$$
I_{\mu \nu}^{\theta}=-g^{2} \int \frac{d^{3} q}{(2 \pi)^{3}} \frac{\operatorname{tr}\left\{\gamma_{\mu}[M+i(\not q+\not p)] \gamma_{\nu}[M+i \not q]\right\}}{\left[M^{2}+(q+p)^{2}\right]\left(M^{2}+q^{2}\right)} \mathrm{e}^{i \theta(\vec{p} \times \vec{q})}
$$


Here one can follow the same steps as in the case of fundamental fermions, leading to

$$
\begin{aligned}
I_{\mu \nu}^{\theta}= & -2 g^{2} \int_{0}^{1} d x \int \frac{d^{3} q}{(2 \pi)^{3}} \\
& \times \frac{M \epsilon_{\mu \nu \alpha} p_{\alpha}-2 q_{\mu \nu}+2 x(1-x) p_{\mu} p_{\nu}+\left[M^{2}+q^{2}-x(1-x) p^{2}\right] \delta_{\mu \nu}}{\left[q^{2}+M^{2}+x(1-x) p^{2}\right]^{2}} \mathrm{e}^{i \theta(\vec{p} \times \vec{q})} .
\end{aligned}
$$

Once more, by using (B.8)-(B.10) one readily finds the expressions (3.20).

To conclude, we outline the calculation of the function $\mathcal{A}\left(p_{1}, p_{2}, \theta\right)_{\mathrm{NP}}$ in Eq. (3.21). As in the case of the two point function, the only difference with respect to the planar part analyzed in the previous subsection [cf. Eq. (B.6)] is the presence of the noncommutative phase. This yields

$$
\begin{aligned}
\mathcal{A}\left(p_{1}, p_{2}, \theta\right)_{\mathrm{NP}}= & 4 M g^{3} \int \frac{d^{3} q}{(2 \pi)^{3}} \int_{0}^{1} d x_{1} \int_{0}^{1-x_{2}} d x_{2} \\
& \times \frac{M^{2}+q^{2}+\left(p_{1}+p_{2}\right) \cdot q}{\left\{\left[q+\left(x_{1}+x_{2}\right) p_{1}+x_{2} p_{2}\right]^{2}+\Delta\right\}^{3}} \mathcal{W}\left(p_{1}, p_{2}, q\right)_{\mathrm{NP}}
\end{aligned}
$$

where the phases are given in Eq. (3.23). Because of the structure of the noncommutative phases, $\mathcal{A}\left(p_{1}, p_{2}, \theta\right)_{\mathrm{NP}}$ is indeed a sum of terms of the form (3.24). After shifting the loop momentum, the integral can be evaluated with the help of Eq. (B.9) together with

$$
\begin{aligned}
& \int \frac{d^{3} q}{(2 \pi)^{3}} \frac{q_{\mu} \mathrm{e}^{i \theta(\vec{p} \times \vec{q})}}{\left(q^{2}+\omega^{2}\right)^{3}}=\frac{i}{32 \pi} \frac{\theta(p)_{\mu}}{\theta(p)^{2}} \sqrt{\frac{2}{\pi}}|\omega|^{-\frac{1}{2}}(\theta|\vec{p}|)^{\frac{5}{2}} K_{\frac{1}{2}}(\theta|\vec{p}||\omega|), \\
& \int \frac{d^{3} q}{(2 \pi)^{3}} \frac{\mathrm{e}^{i \theta(\vec{p} \times \vec{q})}}{\left(q^{2}+\omega^{2}\right)^{3}}=\frac{1}{32 \pi} \sqrt{\frac{2}{\pi}}(\theta|\vec{p}|)^{\frac{3}{2}}|\omega|^{-\frac{3}{2}} K_{\frac{3}{2}}(\theta|\vec{p}||\omega|) .
\end{aligned}
$$

In our case the constant $\omega$ is replaced by $\Delta$. Thus, for large values of the fermion mass and at fixed incoming momenta the argument of the Bessel functions is very large and the corresponding integrals vanish exponentially. In the same way, if $M \rightarrow 0$ at finite momenta $\Delta$ is nonzero and the corresponding integrals remain finite. Thus, because of the presence of an overall power of $M$ in front of (B.13), all the integrals contributing to the function $\mathcal{A}\left(p_{1}, p_{2}, \theta\right)_{\mathrm{NP}}$ will vanish in that limit.

\section{References}

[1] M. R. Douglas and N. A. Nekrasov, "Noncommutative field theory," Rev. Mod. Phys. 73, 977 (2001) [hep-th/0106048].

[2] R. J. Szabo, "Quantum field theory on noncommutative spaces," [hep-th/0109162].

[3] M. M. Sheikh-Jabbari, "Open strings in a B-field background as electric dipoles," Phys. Lett. B 455, 129 (1999) [hep-th/9901080].

D. Bigatti and L. Susskind, "Magnetic fields, branes and noncommutative geometry," Phys. Rev. D 62, 066004 (2000) [hep-th/9908056].

[4] L. Susskind, "The quantum Hall fluid and non-commutative Chern Simons theory," hep-th/0101029. 
[5] A. P. Polychronakos, "Quantum Hall states as matrix Chern-Simons theory," J. High Energy Phys. 0104, 011 (2001) [hep-th/0103013].

E. Fradkin, V. Jejjala and R. G. Leigh, "Non-commutative Chern-Simons for the Quantum Hall System and Duality," cond-mat/0205653.

[6] S. Hellerman and M. Van Raamsdonk, "Quantum Hall physics equals noncommutative field theory," J. High Energy Phys. 0110, 039 (2001) [hep-th/0103179].

[7] J. L. Barbón and A. Paredes, "Noncommutative field theory and the dynamics of quantum Hall fluids," Int. J. Mod. Phys. A 17, 3589 (2002) [hep-th/0112185].

[8] A. Connes, M. R. Douglas and A. Schwarz, "Noncommutative geometry and matrix theory: Compactification on tori," J. High Energy Phys. 9802, 003 (1998) [hep-th/9711162].

[9] H. Aoki, N. Ishibashi, S. Iso, H. Kawai, Y. Kitazawa and T. Tada, "Noncommutative Yang-Mills in IIB matrix model," Nucl. Phys. B 565 (2000) 176 [hep-th/9908141].

[10] I. Bars and D. Minic, "Non-commutative geometry on a discrete periodic lattice and gauge theory," Phys. Rev. D 62 (2000) 105018 [hep-th/9910091].

[11] J. Ambjørn, Y. M. Makeenko, J. Nishimura and R. J. Szabo, "Finite N matrix models of noncommutative gauge theory," J. High Energy Phys. 9911, 029 (1999) [hep-th/9911041].

[12] J. Ambjørn, Y. M. Makeenko, J. Nishimura and R. J. Szabo, "Nonperturbative dynamics of noncommutative gauge theory," Phys. Lett. B 480, 399 (2000) [hep-th/0002158].

[13] J. Ambjørn, Y. M. Makeenko, J. Nishimura and R. J. Szabo, "Lattice gauge fields and discrete noncommutative Yang-Mills theory," J. High Energy Phys. 0005, 023 (2000) [hep-th/0004147].

[14] A. González-Arroyo and M. Okawa, "The Twisted Eguchi-Kawai Model: A Reduced Model For Large N Lattice Gauge Theory," Phys. Rev. D 27, 2397 (1983).

[15] W. Bietenholz, F. Hofheinz and J. Nishimura, "A non-perturbative study of gauge theory on a non-commutative plane," J. High Energy Phys. 0209, 009 (2002) [hep-th/0203151].

[16] W. Bietenholz, F. Hofheinz and J. Nishimura, "Simulating non-commutative field theory," hep-lat/0209021.

[17] S. Profumo, "Noncommutative Principal Chiral Models," J. High Energy Phys. 0205, 014 (2002) [hep-th/0111285].

[18] J. Ambjørn and S. Catterall, "Stripes from (noncommutative) stars," hep-lat/0209106.

[19] S. S. Gubser and S. L. Sondhi, "Phase structure of non-commutative scalar field theories," Nucl. Phys. B 605 (2001) 395 [hep-th/0006119].

[20] J. Nishimura and M. A. Vázquez-Mozo, "Noncommutative chiral gauge theories on the lattice with manifest star-gauge invariance," J. High Energy Phys. 0108, 033 (2001) [hep-th/0107110].

[21] A. Coste and M. Lüscher, "Parity Anomaly And Fermion Boson Transmutation In Three-Dimensional Lattice QED," Nucl. Phys. B 323, 631 (1989).

[22] S. Minwalla, M. Van Raamsdonk and N. Seiberg, "Noncommutative perturbative dynamics," J. High Energy Phys. 0002, 020 (2000) [hep-th/9912072]. 
[23] C. S. Chu, "Induced Chern-Simons and WZW action in noncommutative spacetime," Nucl. Phys. B 580, 352 (2000) [hep-th/0003007].

[24] W. Bietenholz and J. Nishimura, "Ginsparg-Wilson fermions in odd dimensions," J. High Energy Phys. 0107 (2001) 015, [hep-lat/0012020];

W. Bietenholz, J. Nishimura and P. Sodano, "Chern-Simons theory on the lattice," [hep-lat/0207010].

[25] A. P. Polychronakos, "Quantum Hall states on the cylinder as unitary matrix Chern-Simons theory," J. High Energy Phys. 0106, 070 (2001) [hep-th/0106011].

[26] Y. Makeenko, "Reduced models and noncommutative gauge theories," JETP Lett. 72 (2000) 393 [hep-th/0009028].

[27] R. J. Szabo, "Discrete noncommutative gauge theory," Mod. Phys. Lett. A 16, 367 (2001) [hep-th/0101216].

[28] Y. Makeenko, Methods of Contemporary Gauge Theories, Cambridge 2002.

[29] H. Kawai, R. Nakayama and K. Seo, "Comparison Of The Lattice Lambda Parameter With The Continuum Lambda Parameter In Massless QCD," Nucl. Phys. B 189, 40 (1981).

[30] M. M. Sheikh-Jabbari, "Renormalizability of the supersymmetric Yang-Mills theories on the noncommutative torus," J. High Energy Phys. 9906, 015 (1999) [hep-th/9903107].

A. Armoni, "Comments on perturbative dynamics of non-commutative Yang-Mills theory," Nucl. Phys. B 593, 229 (2001) [hep-th/0005208].

[31] I. Montvay and G. Münster, Quantum Fields on the Lattice, Cambridge 1994.

[32] A. N. Redlich, "Gauge Noninvariance And Parity Nonconservation Of Three-Dimensional Fermions," Phys. Rev. Lett. 52, 18 (1984).

A. N. Redlich, "Parity Violation And Gauge Noninvariance Of The Effective Gauge Field Action In Three-Dimensions," Phys. Rev. D 29, 2366 (1984).

[33] A. J. Niemi and G. W. Semenoff, "Axial Anomaly Induced Fermion Fractionization And Effective Gauge Theory Actions In Odd Dimensional Space-Times," Phys. Rev. Lett. 51, 2077 (1983).

L. Alvarez-Gaumé, S. Della Pietra and G. W. Moore, "Anomalies And Odd Dimensions," Annals Phys. 163, 288 (1985).

A. N. Redlich and L. C. Wijewardhana, "Induced Chern-Simons Terms At High Temperatures And Finite Densities," Phys. Rev. Lett. 54, 970 (1985).

S. Deser, L. Griguolo and D. Seminara, "Gauge invariance, finite temperature and parity anomaly in $D=3$," Phys. Rev. Lett. 79, 1976 (1997) [hep-th/9705052].

[34] G. V. Dunne, "Aspects of Chern-Simons theory," hep-th/9902115.

[35] D. Khveshchenko and P. B. Wiegmann, "Physical Realization Of The Parity Anomaly And Quantum Hall Effect," Phys. Lett. B 225 (1989) 279.

[36] L. Alvarez-Gaumé and E. Witten, "Gravitational Anomalies," Nucl. Phys. B 234, 269 (1984).

[37] E. Witten, "An SU(2) Anomaly," Phys. Lett. B 117, 324 (1982). 
[38] T. Reisz, "A Power Counting Theorem For Feynman Integrals On The Lattice," Commun. Math. Phys. 116, 81 (1988).

T. Reisz, "A Convergence Theorem For Lattice Feynman Integrals With Massless Propagators," Commun. Math. Phys. 116, 573 (1988).

[39] A. González-Arroyo and C. P. Korthals Altes, "Reduced Model For Large N Continuum Field Theories," Phys. Lett. B 131, 396 (1983).

[40] T. Filk, "Divergencies In A Field Theory On Quantum Space," Phys. Lett. B 376 (1996) 53.

[41] N. Ishibashi, S. Iso, H. Kawai and Y. Kitazawa, "Wilson loops in noncommutative Yang-Mills," Nucl. Phys. B 573 (2000) 573 [hep-th/9910004].

[42] F. Ardalan and N. Sadooghi, "Anomaly and nonplanar diagrams in noncommutative gauge theories," Int. J. Mod. Phys. A 17, 123 (2002) [hep-th/0009233].

K. A. Intriligator and J. Kumar, "*-wars episode I: The phantom anomaly," Nucl. Phys. B 620, 315 (2002) [hep-th/0107199].

L. Bonora and A. Sorin, "Chiral anomalies in noncommutative YM theories," Phys. Lett. B 521, 421 (2001) [hep-th/0109204].

C. P. Martin, "The covariant form of the gauge anomaly on noncommutative $\mathbb{R}^{2 n}$," Nucl. Phys. B 623, 150 (2002) [hep-th/0110046].

[43] A. Armoni, E. Lopez and S. Theisen, "Nonplanar anomalies in noncommutative theories and the Green-Schwarz mechanism," J. High Energy Phys. 0206, 050 (2002) [hep-th/0203165].

[44] G. Arcioni and M. A. Vázquez-Mozo, "Thermal effects in perturbative noncommutative gauge theories," J. High Energy Phys. 0001, 028 (2000) [hep-th/9912140].

[45] P. Ginsparg and K. Wilson, "A remnant of chiral symmetry on the lattice", Phys. Rev. D25 (1982) 2649.

[46] M. Lüscher, "Exact chiral symmetry on the lattice and the Ginsparg-Wilson relation", Phys. Lett. B428 (1998) 342, hep-lat/9802011.

[47] R. Narayanan and H. Neuberger, "A construction of lattice chiral gauge theories", Nucl. Phys. B443 (1995) 305, hep-th/9411108.

[48] H. Neuberger, "Exactly massless quarks on the lattice" Phys. Lett. 417 (1998) 141, hep-lat/9707022; "More about exactly massless quarks on the lattice", Phys. Lett. 427 (1998) 353, hep-lat/9801031.

[49] H. Aoki, S. Iso and K. Nagao, "Ginsparg-Wilson Relation, Topological Invariants and Finite Noncommutative Geometry," hep-th/0209223.

[50] Y. Kikukawa and H. Neuberger, "Overlap in odd dimensions," Nucl. Phys. B 513 (1998) 735 [hep-lat/9707016].

[51] R. Narayanan and J. Nishimura, "Parity-invariant lattice regularization of a three-dimensional gauge-fermion system," Nucl. Phys. B 508 (1997) 371 [hep-th/9703109].

[52] V. P. Nair and A. P. Polychronakos, "On level quantization for the noncommutative Chern-Simons theory," Phys. Rev. Lett. 87, 030403 (2001) [hep-th/0102181].

D. Bak, K. M. Lee and J. H. Park, "Chern-Simons theories on noncommutative plane," Phys. Rev. Lett. 87, 030402 (2001) [hep-th/0102188]. 
[53] M. M. Sheikh-Jabbari, "A note on noncommutative Chern-Simons theories," Phys. Lett. B 510, 247 (2001) [hep-th/0102092].

[54] E. F. Moreno and F. A. Schaposnik, "The Wess-Zumino-Witten term in non-commutative two-dimensional fermion models," J. High Energy Phys. 0003, 032 (2000) [hep-th/0002236]. E. F. Moreno and F. A. Schaposnik, "Wess-Zumino-Witten and fermion models in noncommutative space," Nucl. Phys. B 596, 439 (2001) [hep-th/0008118].

[55] A. H. Chamseddine and J. Fröhlich, "The Chern-Simons Action In Noncommutative Geometry," J. Math. Phys. 35, 5195 (1994) [hep-th/9406013].

G. H. Chen and Y. S. Wu, "One-loop shift in noncommutative Chern-Simons coupling," Nucl. Phys. B 593, 562 (2001) [hep-th/0006114].

N. Grandi and G. A. Silva, "Chern-Simons action in noncommutative space," Phys. Lett. B 507, 345 (2001) [hep-th/0010113].

A. P. Polychronakos, "Noncommutative Chern-Simons terms and the noncommutative vacuum," J. High Energy Phys. 0011, 008 (2000) [hep-th/0010264].

J. Kluson, "Matrix model and noncommutative Chern-Simons theory," Phys. Lett. B 505, 243 (2001) [hep-th/0012184].

A. K. Das and M. M. Sheikh-Jabbari, "Absence of higher order corrections to noncommutative Chern-Simons coupling," J. High Energy Phys. 0106, 028 (2001) [hep-th/0103139].

C. P. Martin, "Computing noncommutative Chern-Simons theory radiative corrections on the back of an envelope," Phys. Lett. B 515, 185 (2001) [hep-th/0104091].

[56] J. Kiskis, R. Narayanan and H. Neuberger, "Proposal for the numerical solution of planar QCD," Phys. Rev. D 66 (2002) 025019 [hep-lat/0203005].

[57] Y. Kikukawa and H. Suzuki, "Chiral anomalies in the reduced model," J. High Energy Phys. 0209 (2002) 032 [hep-lat/0207009].

[58] H. Aoki, S. Iso and K. Nagao, "Chiral anomaly on fuzzy 2-sphere," hep-th/0209137. 